\title{
A pedagogical walkthrough of computational modeling and simulation of Wnt signaling pathway using static causal models in MATLAB
}

\author{
Shriprakash Sinha
}

\begin{abstract}
Simulation study in systems biology involving computational experiments dealing with Wnt signaling pathways abound in literature but often lack a pedagogical perspective that might ease the understanding of beginner students and researchers in transition, who intend to work on the modeling of the pathway. This paucity might happen due to restrictive business policies which enforce an unwanted embargo on the sharing of important scientific knowledge. A tutorial introduction to computational modeling of Wnt signaling pathway in a human colorectal cancer dataset using static Bayesian network models is provided. The walkthrough might aid biologists/informaticians in understanding the design of computational experiments that is interleaved with exposition of the MATLAB code and causal models from Bayesian network toolbox. The manuscript elucidates the coding contents of the advance article by Sinha (Integr. Biol. 6:1034-1048, 2014) and takes the reader in a step-by-step process of how (a) the collection and the transformation of the available biological information from literature is done, (b) the integration of the heterogeneous data and prior biological knowledge in the network is achieved, (c) the simulation study is designed, (d) the hypothesis regarding a biological phenomena is transformed into computational framework, and (e) results and inferences drawn using $d$-connectivity/separability are reported. The manuscript finally ends with a programming assignment to help the readers get hands-on experience of a perturbation project. Description of MATLAB files is made available under GNU GPL v3 license at the Google code project on https://code. google.com/p/static-bn-for-wnt-signaling-pathway and https://sites.google.com/site/shriprakashsinha/ shriprakashsinha/projects/static-bn-for-wnt-signaling-pathway. Latest updates can be found in the latter website.
\end{abstract}

Keywords: Wnt signaling pathway, Bayesian network, Prior biological knowledge, Epigenetic information, Heterogeneous data integration, Hypothesis testing, Inference

\section{Introduction}

A tutorial introduction to computational modeling of Wnt signaling pathway in a human colorectal cancer dataset using static Bayesian network models is provided. This work endeavors to expound in detail the simulation study in MATLAB along with the code while explaining the concepts related to Bayesian networks. This is done in order to ease the understanding of beginner students and researchers in transition to computational signaling biology, who intend to work in the field of modeling of the

Correspondence: sinha.shriprakash@yandex.com

104-Madhurisha Heights Phase 1, Risali 490006 Bhilai, India signaling pathways. The manuscript elucidates (a) embedding of prior biological knowledge, (b) integration of heterogeneous information, (c) transformation of biological hypothesis into computational framework, and (d) design of the experiments, in a simple manner. This is interleaved with aspects of Bayesian network toolbox and MATLAB code so as to help readers get a feel of a project related to modeling of the pathway. Programming along with the exposition in the manuscript could clear up issues faced during the execution of the project.

This manuscript uses the contents of the advance article [1] as a basis to explain the workflow of a computational simulation project involving Wnt signaling pathway in 
human colorectal cancer (See Table 2 and Fig. 1 for description). The aim of [1] was to computationally test whether the activation of $\beta$-catenin and TCF4-based transcription complex always corresponds to the tumorous state of the test sample or not. To achieve this, the gene expression data provided by [2] was used in the computational experiments. Furthermore, to refine the model, prior biological knowledge related to the intra/extracellular factors of the pathway (available in literature) was integrated along with epigenetic information. Section 4 of [1] has been reproduced for completeness in Tables 1, 2, 3, 4, 5, 6, and 7 in order. These tables provide introductory theory that will help in understanding the various aspects of the MATLAB code for modeling and simulation experiments that are explained later. More specifically, Table 1 gives an introduction to Bayesian networks. Tables 2 and 3 give a brief introduction to the canonical Wnt signaling pathway and the involved epigenetic factors, respectively. Table 4 gives a description of the three Bayesian network models developed with(out) prior biological knowledge. Tables 5 and 6 develop the network models with epigenetic information along with biological knowledge (Tables 8 and 9). Finally, Table 7 discusses a network model that has negligible prior biological knowledge. Code will be presented in typewriter font and functions in the text will be presented in sans serif. Reasons for taking certain approach and important information within the project are presented in small capitals.

\section{Motivation}

\subsection{The project and issues involved}

Drafting a manuscript that contains a pedagogical outlook of all the theory and the MATLAB code is a challenging task. This is because the background work of coding in a modeling and simulation project faces several issues that need to be overcome. Here, a few of these issues are discussed, but they are by no means complete. Some of the issues might be general across different computational biology projects while others might be more specific to the current project.

The advanced article of [1] contains three different network models, one of which is the naive Bayes model. The implemented naive Bayes model in [1] is a simplification of the primitive model proposed in [3]. The other two models are improvements over the naive Bayes model which incorporate prior biological knowledge. This manuscript describes the implementation of these models using a single colorectal cancer dataset. The reason for doing this was to test the effectiveness of incorporating prior biological knowledge gleaned from literature study of genes related to the dataset as well as test a biological hypothesis from a computational point of view. The main issues that one faces in this project are (a) finding biological causal relations from already published wet lab experiments, (b) designing the graphical network from biological knowledge, (c) translating the measurements into numerical values that form the prior beliefs of nodes in the network, (d) estimating the conditional probability values for nodes with parents, (e) framing the biological hypothesis into computational framework, (f) choosing the design of the learning experiment depending on the type of data, $(\mathrm{g})$ inferring the hidden biological relations after the execution of the Bayesian network inference engine, and finally (h) presenting the results in a proper format via statistical significance tests.

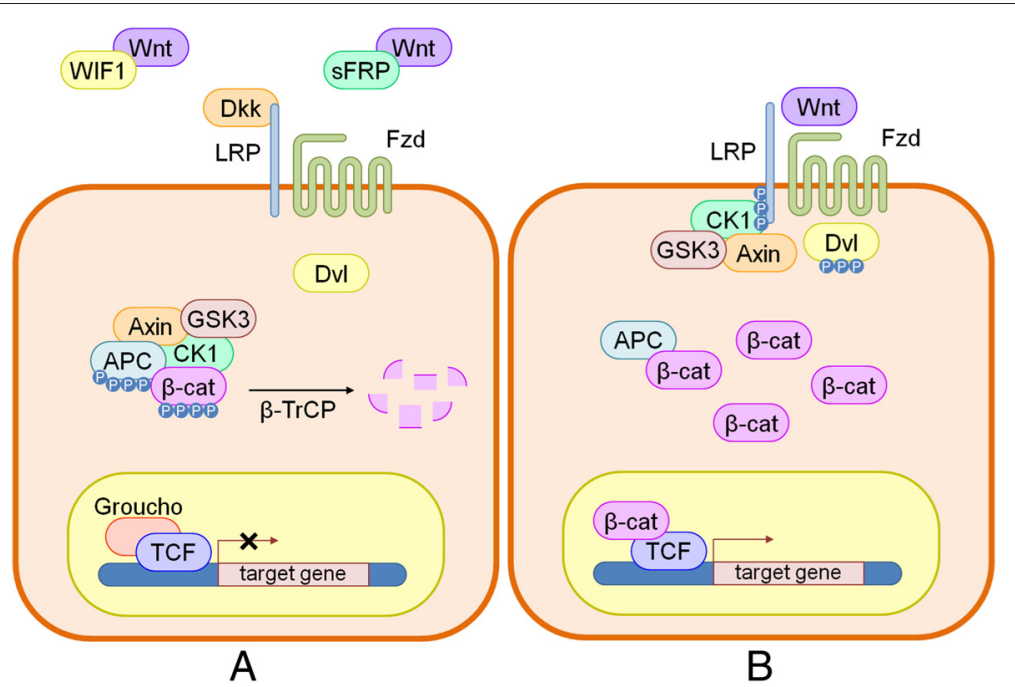

Fig. 1 A cartoon of Wnt signaling pathway contributed by [3]. Part a represents the destruction of $\beta$-catenin leading to the inactivation of the Wnt target gene. Part $\mathbf{b}$ represents activation of Wnt target gene 
Table 1 Bayesian networks from [1]

Bayesian networks. In reverse engineering methods for control networks [10] there exist many methods that help in the construction of the networks from the datasets as well as give the ability to infer causal relations between components of the system. A widely known architecture among these methods is the Bayesian network (BN). These networks can be used for causal reasoning or diagnostic reasoning or both. It has been shown through reasoning and examples in [11] that the probabilistic inference mechanism applied via Bayesian networks are analogous to the structural equation modeling in path analysis problems. Initial works on BNs in $[12,13]$ suggest that the networks only need a relatively small amount of marginal probabilities for nodes that have no incoming arcs and a set of conditional probabilities for each node having one or more incoming arcs. The nodes form the driving components of a network and the arcs define the interactive influences that drive a particular process. Under these assumptions of influences the joint probability distribution of the whole network or a part of it can be obtained via a special factorization that uses the concept of direct influence and through dependence rules that define d-connectivity/separability as mentioned in [14] and [15]. This is illustrated through a simple example in [1 1].

The Bayesian networks work by estimating the posterior probability of the model given the dataset. This estimation is usually referred to as the Bayesian score of the model conditioned on the dataset. Mathematically, let $\mathcal{S}$ represent the model given the data $\mathcal{D}$ and $\xi$ is the background knowledge. Then according to the Bayes Theorem [16]:

$$
\begin{aligned}
\mathcal{P}(\mathcal{S} \mid \mathcal{D}, \xi) & =\frac{\mathcal{P}(\mathcal{S} \cap \mathcal{D} \mid \xi)}{\mathcal{P}(\mathcal{D} \mid \xi)} \\
& =\frac{\mathcal{P}(\mathcal{S} \mid \xi) \times \mathcal{P}(\mathcal{D} \mid \mathcal{S}, \xi)}{\mathcal{P}(\mathcal{D} \mid \xi)} \\
\text { posterior } & =\frac{\text { prior } \times \text { likelihood }}{\text { constant }}
\end{aligned}
$$

Thus the Bayesian score is computed by evaluating the posterior distribution $\mathcal{P}(\mathcal{S} \mid \mathcal{D}, \xi)$ which is proportional to the prior distribution of the model $\mathcal{P}(\mathcal{S} \mid \xi)$ and the likelihood of the data given the model $\mathcal{P}(\mathcal{D} \mid \mathcal{S}, \xi)$. It must be noted that the background knowledge is assumed to be independent of the data. Next, since the evaluation of probabilities require multiplications a simpler way is to take logarithmic scores which boils down to addition. Thus, the estimation takes the form

$$
\begin{aligned}
\log \mathcal{P}(\mathcal{S} \mid \mathcal{D}, \xi)= & \log \mathcal{P}(\mathcal{S} \mid \xi)+\log \mathcal{P}(\mathcal{D} \mid \mathcal{S}, \xi) \\
& -\log \mathcal{P}(\mathcal{D} \mid \xi) \\
= & \log \mathcal{P}(\mathcal{S} \mid \xi)+\log \mathcal{P}(\mathcal{D} \mid \mathcal{S}, \xi) \\
& + \text { constant }
\end{aligned}
$$

Finally, the likelihood of the function can be evaluated by averaging over all possible local conditional distributions parameterized by $\theta_{i}$ s that depict the conditioning of parents. This is equated via

$$
\begin{aligned}
\mathcal{P}(\mathcal{D} \mid \mathcal{S}, \xi) & =\int_{\theta_{1}} \cdots \int_{\theta_{n}} \mathcal{P}\left(\mathcal{D}, \theta_{i} \mid \mathcal{S}\right) d \theta_{i} \\
& =\int_{\theta_{1}} \cdots \int_{\theta_{n}} \mathcal{P}\left(\mathcal{D} \mid \theta_{i} \mathcal{S}\right) \mathcal{P}\left(\theta_{i} \mid \mathcal{S}\right) d \theta_{i}
\end{aligned}
$$

Work on biological systems that make use of Bayesian networks can also be found in [17-21]. Bayesian networks are good in generating network structures and testing a targeted hypothesis which confine the experimenter to derive causal inferences [22]. But a major disadvantage of the Bayesian networks is that they rely heavily on the conditional probability distributions which require good sampling of datasets and are computationally intensive. On the other hand, these networks are quite robust to the existence of the unobserved variables and accommodate noisy datasets. They also have the ability to combine heterogeneous datasets that incorporate different modalities. In this work, simple static Bayesian network models have been developed with an aim to show how (a) incorporation of heterogeneous data can be done to increase prediction accuracy of test samples, (b) prior biological knowledge can be embedded to model biological phenomena behind the Wnt pathway in colorectal cancer, (c) to test the hypothesis regarding direct correspondence of active state of $\beta$-catenin-based transcription complex and the state of the test sample via segregation of nodes in the directed acyclic graphs of the proposed models, and (d) inferences can be made regarding the hidden biological relationships between a particular gene and the $\beta$-catenin transcription complex. This work uses MATLAB-implemented BN toolbox from [4].

Table 2 Canonical Wnt pathway from [1]

Canonical Wnt signaling pathway. The canonical Wnt signaling pathway is a transduction mechanism that contributes to embryo development and controls homeostatic self-renewal in several tissues [8]. Somatic mutations in the pathway are known to be associated with cancer in different parts of the human body. Prominent among them is the colorectal cancer case [23]. In a succinct overview, the Wnt signaling pathway works when the Wnt ligand gets attached to the frizzled $(f z d) / L R P$ coreceptor complex. Fzd may interact with the disheveled (Dv/) causing phosphorylation. It is also thought that Wnts cause phosphorylation of the LRP via casein kinase $1(C K 1)$ and kinase GSK3. These developments further lead to attraction of axin which causes inhibition of the formation of the degradation complex. The degradation complex constitutes of axin, the $\beta$-catenin transportation complex APC, CK1, and GSK3. When the pathway is active, the dissolution of the degradation complex leads to stabilization in the concentration of $\beta$-catenin in the cytoplasm. As $\beta$-catenin enters into the nucleus, it displaces the Groucho and binds with transcription cell factor TCF, thus instigating transcription of Wnt target genes. Groucho acts as lock on TCF and prevents the transcription of target genes which may induce cancer. In cases when the Wnt ligands are not captured by the coreceptor at the cell membrane, axin helps in the formation of the degradation complex. The degradation complex phosphorylates $\beta$-catenin which is then recognized by Fbox/WD repeat protein $\beta-\operatorname{TrCP} . \beta-\operatorname{TrCP}$ is a component of ubiquitin ligase complex that helps in ubiquitination of $\beta$-catenin, thus marking it for degradation via the proteasome. Cartoons depicting the phenomena of Wnt activation are shown in Fig. 1a, b, respectively. 
Table 3 Epigenetic factors from [1]

Epigenetic factors. One of the widely studied epigenetic factors is methylation [24-26]. Its occurrence leads to decrease in the gene expression which affects the working of Wnt signaling pathways. Such characteristic trends of gene silencing like that of secreted frizzled-related proteins (SFRP) family in nearly all human colorectal tumor samples have been found at extracellular level [27]. Similarly, methylation of genes in the Dickkopf (DKKx [28, 29]), Dapper antagonist of catenin (DACTx [2]), and Wnt inhibitory factor-1 (WIF1 [30]) family are known to have a significant effect on the Wnt pathway. Also, histone modifications (a class of proteins that help in the formation of chromatin which packs the DNA in a special form [31]) can affect gene expression [32]. In the context of the Wnt signaling pathway, it has been found that DACT gene family shows a peculiar behavior in colorectal cancer [2]. DACT1 and DACT2 showed repression in tumor samples due to increased methylation while DACT3 did not show obvious changes to the interventions. It is indicated that DACT3 promoter is simultaneously modified by both the repressive and activating (bivalent) histone modifications ([2]).

\subsection{Biological causal relations}

Often, biological causal relations are embedded in the literature pertaining to wet lab experiments in molecular biology. These relations manifest themselves as discovery/confirmation of one or multiple factors affecting the expression of a gene by either inhibiting or activating it. In context of the dataset used in the current work, the known causal relations were gleaned from review of such literature for each intra/extracellular factor involved in the pathway. The arcs in the Bayesian networks with prior biological knowledge encode these causal semantics. For those factors whose relations have not been confirmed but known to be involved in the pathway, the causal arcs were segregated via a latent variable that is introduced into the Bayesian network. The latent variable in the form of "sample" (see Fig. 2) is extremely valuable as it connects the factors whose relations have not been confirmed till now, to factors whose influences have been confirmed in the pathway. Detailed explanation of the connectivity can be found in Table 6. Also, the introduction of latent variable in a causal model opens an avenue to assume the presence of measurements that haven't been recorded. Intuitively, for cancer samples the hidden measurements might be different from those for normal samples. The connectivity of factors through the variable provides an important route to infer biological relations. Finally, the problem with such models is that it is static in nature. This means that the models represent only a snapshot of the connectivity in time, which is still an important information for further research. By using time course data it might be possible to reveal greater biological information dynamically. The current work lacks in this endeavor and considers the introduction of time course-based dynamic models for future research work.

\subsection{Bayesian networks, parameter estimation, biological hypothesis}

Bayesian networks are probabilistic graphical models that encode causal semantics among various factors using arcs and nodes. The entire network can represent a framework for a biological pathway and can be used to predict, explore or explain certain behaviors related to the pathway (See Tables 5 and 6 and Fig. 3 for description). As previously stated, the directionality of the arcs define the causal influence while the nodes represent the involved factors. Also, it is not just the arcs and nodes that play a crucial role. Information regarding the strength of the belief in a factor's involvement is encoded as prior probability (priors) or conditional probability values. Estimation of these probabilities are either via expert's knowledge or numerical estimations in the form of frequencies gleaned from measurements provided in the literature from wet lab experiments. In this project, the nodes are discrete in nature. Since the models are a snapshot in time, discrete nodes help in encoding specific behavior in time. Here, discretization means defining the states in which a factor can be (say a gene expression is on or off, or methylation is on or off, etc). As stated above, this leads to loss of continuous information revealed in time series data.

As depicted in the model in Fig. 2 and described in Tables 5 and 6 , to test one of the biological hypothesis that TRCMPLX is not always switched on (off) when the sample is tumorous (normal), the segregation of TRCM$P L X$ node from Sample node was made in [1]. Primitive models of the Naive Bayes network assume direct correspondence of TRCMPLX and Sample as depicted in [1] and [3]. The segregated design helps in framing the biological hypothesis into computational framework. The basic factor in framing the biological hypothesis to

Table 4 Bayesian Wnt pathway from [1]

Bayesian Wnt pathway. Three static models have been developed based on particular gene set measured for human colorectal cancer cases [2]. Available epigenetic data for individual gene is also recorded. For sake of simplicity, the models are connoted as $\mathcal{M}_{\mathrm{PBK}+\mathrm{El}}$ (model with prior biological knowledge (PBK) and epigenetic information (EI)), $\mathcal{M}_{\text {PBK }}$ (model with PBK only), and $\mathcal{M}_{\text {NB+MPBK }}$ (model with naive Bayes (NB) formulation and minimal PBK). All models are simple directed acyclic graphs (DAG) with nodes and edges. Figure 2 shows a detailed influence diagram of $\mathcal{M}$ PBK+El between the nodes and the edges. The nodes specify status of gene expression (DKK1, DKK2, DKK3-1, DKK3-2, DKK4, DACT1, DACT2, DACT3, SFRP1, SFRP2, SFRP3, SFRP4, SFRP5, WIF1, MYC, CD44, CCND1, and LEF1), methylation (MeDACT1, MeDACT2, MeSFRP1, MeSFRP2, MeSFRP4, MeSFRP5, MeDKK1, MeDKK4, and MeWIF1), histone marks for DACT3 (H3K27me3 and H3K4me3), transcription complex TRCMPLX, samples Sample and factors involved in formation of TRCMPLX like $\beta$-catenin, TCF4, and LEF1. Note that there were two recordings of gene expression DKK3 and thus were distinguished by DKK3 - 1 and DKK3 - 2 . Some causal relations are based on prior biological knowledge and others are based on assumptions, elucidation of which follows in the next section. 
Table 5 Network with PBK+El from [1]

Network with PBK and El the NB model [3] assumes that the activation (inactivation) of $\beta$-catenin-based transcription complex is equivalent to the fact that the sample is cancerous (normal). This assumption needs to be tested and in this research work, the two newly improvised models based on prior biological knowledge regarding the signaling pathway assume that sample prediction may not always mean that the $\beta$-catenin-based transcription complex is activated. These assumptions are incorporated by inserting another node of Sample for which gene expression measurements were available. This is separate from the TRCMPLX node that influences a particular set of known genes in the human colorectal cancer. For those genes whose relation with the TRCMPLX is currently not known or biologically affirmed, indirect paths through the Sample node to the TRCMPLX exist, technical aspect of which will be described shortly. Since all gene expressions have been measured from a sample of subjects, the expression of genes is conditional on the state of the Sample. Here, both tumorous and normal cases are present in equal amounts. The transcription factor TRCMPLX under investigation is known to operate with the help of interaction between $\beta$-catenin with TCF4 and LEF1 [9, 33]. It is also known that the regions in the TSS of MYC [34], CCND1 [35], CD44 [36], SFRP1 [37], WIF1 [38], DKK1 [39], and DKK4 [40, 41] contain factors that have affinity to $\beta$-catenin-based TRCMPLX. Thus, expression of these genes are shown to be influenced by $\operatorname{TRCMPLX}$, in Fig. 2.

Roles of DKK2 [42] and DKK3 $[43,44]$ have been observed in colorectal cancer but their transcriptional relation with $\beta$-catenin-based TRCMPLX is not known. Similarly, SFRP2 is known to be a target of Pax2 transcription factor and yet it affects the $\beta$-catenin Wnt signaling pathway [45]. Similarly, SFRP4 $[46,47]$ and SFRP5 [27] are known to have an effect on the Wnt pathway but their role with TRCMPLX is not well studied. SFRP3 is known to have a different structure and function with respect to the remaining SFRPX gene family [48]. Also, the role of DACT2 is found to be conflicting in the Wnt pathway [49]. Thus, for all these genes whose expression mostly have an extracellular effect on the pathway and information regarding their influence on $\beta$-cateninbased TRCMPLX node is not available, an indirect connection has been made through the Sample node. This connection will be explained at the end of this section.

Table 6 Network with PBK+El continued from [1]

Network with PBK and El continued ... Lastly, it is known that concentration of DVL2 (a member of disheveled family) is inversely regulated by the expression of DACT3 [2]. High DVL2 concentration and suppression of DACT1 leads to increase in stabilization of $\beta$-catenin which is necessary for the Wnt pathway to be active [2]. But in a recent development [7], it has been found that expression of DACT1 positively regulates $\beta$-catenin. Both scenarios need to be checked via inspection of the estimated probability values for $\beta$-catenin using the test data. Thus, there exists direct causal relations between parent nodes DACT1 and DVL2 and child node, $\beta$-catenin. Influence of methylation (yellow hexagonal) nodes to their respective gene (green circular) nodes represent the effect of methylation on genes. Influence of histone modifications in H3K27me3 and H3K4me3 (blue octagonal) nodes to DACT3 gene node represents the effect of histone modification on DACT3. The $\beta$-catenin (blue square) node is influenced by concentration of DVL2 (depending on the expression state of DACT3) and behavior of DACT1. The aforementioned established prior causal biological knowledge is imposed in the BN model with the aim to computationally reveal unknown biological relationships. The influence diagram of this model is shown in Fig. 2 with nodes on methylation and histone modification. Another model $\mathcal{M}_{\text {PBK }}$ (not shown here) was developed excluding the epigenetic information (i.e., removal of nodes depicting methylation and histone modification as well as the influence arcs emerging from them) with the aim to check whether inclusion of epigenetic factors increases the cancer prediction accuracy.

In order to understand indirect connections further, it is imperative to know about $d$-connectivity/separability. In a BN model, this connection is established via the principle of $d$-connectivity which states that nodes are connected in a path when there exists no node in the path that has more than one incoming influence edge or there exists nodes in the path with more than one incoming influence edge which are observed (i.e., evidence regarding such nodes is available) [50]. Conversely, via principle of $d$-separation, nodes are separated in a path when there exists nodes in the path that have more than one incoming influence edge or there exists nodes in the path with at most one incoming influence edge which are observed (i.e., evidence regarding such nodes is available). Figure 3 represents three different cases of connectivity and separation between nodes $\mathcal{A}$ and $\mathcal{C}$ when the path between them passes through node $\mathcal{B}$. Connectivity or dependency exists between nodes $\mathcal{A}$ and $\mathcal{C}$ when (a) evidence is not present regarding node $\mathcal{B}$ in the left graphs of I and II in Fig. 3 or (b) evidence is present regarding node $\mathcal{B}$ in the right graph of III in Fig. 3.

Conversely, separation or independence exists between nodes $\mathcal{A}$ and $\mathcal{C}$ when (a) evidence is present regarding node $\mathcal{B}$ in the right graphs of I and II in Fig. 3 or (b) evidence is not present regarding node $\mathcal{B}$ in the left graph of III in Fig. 3. It would be interesting to know about the behavior of TRCMPLX, given the evidence of state of SFRP3. To reveal such information, paths must exist between these nodes. It can be seen that there are multiple paths between TRCMPLX and SFRP2 in the BN model in Fig. 2. These paths are enumerated as follows:
1. SFRP3, Sample, SFRP1, TRCMPLX
2. SFRP3, Sample, DKK 1, TRCMPLX
3. SFRP3, Sample, WIF1, TRCMPLX
4. SFRP3, sample, CD44, TRCMPLX
5. SFRP3, Sample, DKK4, TRCMPLX
6. SFRP3, Sample, CCND1, TRCMPLX
7. SFRP3, Sample, MYC, TRCMPLX
8. SFRP3, Sample, LEF1, TRCMPLX
9. SFRP3, Sample, DACT3, DVL2, $\beta$-catenin, TRCMPLX
10. SFRP3, Sample, DACT1, $\beta$-catenin, TRCMPLX

Knowledge of evidence regarding nodes of SFRP1 (path 1), DKK1 (path 2), WIF1 (path 3), CD44 (path 4), DKK4 (path 5), CCND1 (path 6), and MYC (path 7) makes Sample and TRCMPLX dependent or d-connected. Further, no evidence regarding state of Sample on these paths instigates dependency or connectivity between SFRP3 and TRCMPLX. On the contrary, evidence regarding LEF1, DACT3, and DACT1 makes Sample (and child nodes influenced by Sample) independent or d-separated from TRCMPLX through paths (8) to (10). Due to the dependency in paths (1) to (7) and the given state of SFRP3 (i.e., evidence regarding it being active or passive), the BN uses these paths during inference to find how TRCMPLX might behave in normal and tumorous test cases. Thus, exploiting the properties of $\mathrm{d}$-connectivity/separability, imposing a biological structure via simple yet important prior causal knowledge and incorporating epigenetic information, BN helps in inferring many of the unknown relation of a certain gene expression and a transcription complex. 
Table 7 Network with NB+MPBK from [1]

Network with minimal PBK. Lastly, a naive Bayes model $\mathcal{M}_{\text {NB+MPBK }}$ with minimal biological knowledge based on [3] model was also developed with an aim to check if the assumed hypothesis that activation state of TRCMPLX is the same as sample being cancerous is correct. In this model, all gene expressions are assumed to be transcribed via the $\beta$-catenin-based TRCMPLX and thus causal arcs exist from TRCMPLX to different gene nodes. The complex itself is influenced by $\beta$-catenin and TCF4 only. Such models can be used for prediction purpose but are not useful in revealing hidden biological relationships as no or minimal prior biological information is imposed on the naive Bayes model. Figure 4 shows the naive Bayes model.

a computational framework requires knowledge of how the known factors of the pathway are involved, how the unknown factors need to be related to the known factors and finally intuitive analysis of the design of the model (for static data). Note that the model is a representation and not complete. Larger datasets will complicate the model and call for more efficient designs.

\subsection{Choice of data}

In a data dependent model, the data guides the working of the model and the results obtained depend on the design of the experiments to be conducted on the data. The current work deals with gene expression data from 24 samples each of human colorectal tumor and matched normal mucosa. Different expression values across the samples are recorded for total of 18 genes known to work at different cellular regions in the pathway. This dataset from [2] was specifically chosen because it covers a small range of important genes whose expression measurements are influenced by epigenetic factors, crucial information about which is enough to build a working

Table 8 Conditional probability tables for nodes (excluding gene expression) of $\mathcal{M}_{\mathrm{PBK}+\mathrm{El}}$

\begin{tabular}{|c|c|c|c|}
\hline \multicolumn{4}{|c|}{ Conditional probability table for nodes } \\
\hline Node & Parents & Cpt values rep. & Node states \\
\hline Sample & - & {$[0.500 .50]^{\top}$} & {$[\mathrm{n} t]$} \\
\hline TCF4 & - & {$[0.100 .90]^{\top}$} & [ia a] \\
\hline DVL2 & DACT3 & {$[0.010 .99 ; 0.990 .01]^{T}$} & {$[\mathrm{lch}$ hc] } \\
\hline \multirow[t]{2}{*}{$\beta$-catenin } & DACT1, & [0.99 0.990 .990 .01 & {$[\mathrm{lch}$ hc] } \\
\hline & DVL2 & $0.010 .010 .010 .99]^{T}$ & \\
\hline \multirow[t]{2}{*}{$\operatorname{TRCMPLX}$} & TCF4, LEF1, & {$\left[0.99^{*}\right.$ ones $(1,7) 0.01$} & [ia a] \\
\hline & $\beta$-catenin & $0.01^{*}$ ones $\left.(1,7) 0.99\right]^{T}$ & \\
\hline MeDACT1 & - & {$[0.83700 .1630]^{T}$} & {$[\mathrm{~nm} \mathrm{~m}]$} \\
\hline MeDACT2 & - & {$[0.33760 .6624]^{T}$} & {$[\mathrm{~nm} \mathrm{~m}]$} \\
\hline MeWIF1 & - & {$[0.16670 .8333]^{T}$} & {$[\mathrm{~nm} \mathrm{~m}]$} \\
\hline MeSFRP1 & - & {$[0.63160 .3684]^{T}$} & {$[\mathrm{~nm} \mathrm{~m}]$} \\
\hline MeSFRP2 & - & {$[0.63160 .3684]^{T}$} & {$[\mathrm{~nm} \mathrm{~m}]$} \\
\hline MeSFRP4 & - & {$\left[\begin{array}{lll}0.8572 & 0.1428\end{array}\right]^{T}$} & {$[\mathrm{~nm} \mathrm{~m}]$} \\
\hline MeSFRP5 & - & {$[0.75000 .2500]^{T}$} & {$[\mathrm{~nm} \mathrm{~m}]$} \\
\hline H3K27me3 & - & {$\left[\begin{array}{lll}0.2391 & 0.7609\end{array}\right]^{T}$} & [ia a $]$ \\
\hline H3K4me3 & - & {$\left[\begin{array}{lll}0.3661 & 0.6339\end{array}\right]^{T}$} & [ia a] \\
\hline
\end{tabular}

Notations in the table mean the following "-" implies no parents exist for the particular node; " $\mathrm{n}$ " - normal, "t" - tumorous, "ia" - inactive, "a" - active, "Ic" - low concentration, "hc" - high concentration, "nm" - non-methylated, "m" - methylation
Table 9 Conditional probability tables for gene nodes of $\mathcal{M}_{\mathrm{PBK}+\mathrm{EI}}$ Conditional probability table for nodes

\begin{tabular}{|c|c|c|}
\hline Node & Parents & Cpt values rep. \\
\hline LEF1 & Sample & {$[0.840 .16 ; 0.160 .84]^{\top}$} \\
\hline \multirow[t]{2}{*}{ MYC } & Sample, & [0.94 0.890 .780 .31 \\
\hline & TRCMPLX & $0.060 .110 .220 .69]^{\top}$ \\
\hline \multirow[t]{2}{*}{ CCND1 } & Sample, & [0.95 0.890 .810 .28 \\
\hline & TRCMPLX & $0.060 .110 .180 .72]^{T}$ \\
\hline \multirow[t]{2}{*}{ CD44 } & Sample, & [0.93 0.90 0.67 0.42; \\
\hline & TRCMPLX & $0.070 .100 .330 .58]^{T}$ \\
\hline \multirow[t]{3}{*}{ DKK1 } & Sample, & [0.95 0.93 0.07 0.05 0.77 0.60 0.40 0.23; \\
\hline & MeDKK1, & 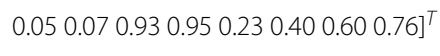 \\
\hline & TRCMPLX & \\
\hline DKK2 & Sample & {$[0.400 .60 ; 0.600 .40]^{\top}$} \\
\hline DKK3-1 & Sample & {$[0.360 .64 ; 0.640 .36]^{\top}$} \\
\hline DKK3-2 & Sample & {$[0.560 .44 ; 0.440 .56]^{\top}$} \\
\hline \multirow[t]{2}{*}{$D K K 4$} & Sample, & [0.94 0.880 .820 .28 \\
\hline & TRCMPLX & $0.060 .110 .180 .72]^{\top}$ \\
\hline \multirow[t]{2}{*}{ DACT1 } & Sample, & {$[0.560 .740 .260 .44$} \\
\hline & MeDACT1 & $0.440 .260 .740 .56]^{\top}$ \\
\hline \multirow[t]{2}{*}{ DACT2 } & Sample, & [0.60 0.71 0.290 .40 \\
\hline & MeDACT2 & $0.400 .290 .710 .60]^{\top}$ \\
\hline \multirow[t]{3}{*}{ DACT3 } & Sample, & [0.88 0.88 0.12 0.88 0.88 0.88 0.12 0.88; \\
\hline & H3K27me3, & 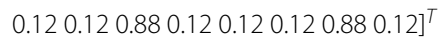 \\
\hline & H3K4me3 & \\
\hline
\end{tabular}

SFRP1 Sample, $\quad[0.880 .980 .020 .120 .200 .960 .040 .80$; MESFRP1， $\quad 0.120 .020 .980 .880 .800 .040 .960 .20]^{\top}$

TRCMPLX

\begin{tabular}{|c|c|c|}
\hline SFRP2 & Sample, & {$\left[\begin{array}{lllllll}0.31 & 0.88 & 0.11 & 0.69 ;\end{array}\right.$} \\
\hline & MeSFRP2 & $0.690 .110 .890 .31]^{T}$ \\
\hline SFRP3 & Sample & {$[0.200 .80 ; 0.800 .20]^{T}$} \\
\hline SFRP4 & Sample, & {$\left[\begin{array}{llllll}0.71 & 0.60 & 0.40 & 0.29 ;\end{array}\right.$} \\
\hline & MeSFRP4 & $0.290 .400 .600 .71]^{\top}$ \\
\hline SFRP5 & Sample, & {$[0.310 .890 .110 .69 ;$} \\
\hline & MeSFRP5 & $0.690 .110 .890 .31]^{T}$ \\
\hline WIF1 & Sample, & [0.96 0.91 0.09 0.04 0.85 0.47 0.56 0.15; \\
\hline & MeWIF1, & $0.040 .090 .910 .960 .150 .530 .470 .85]^{T}$ \\
\hline & TRCMPLX & \\
\hline
\end{tabular}

The state of the gene nodes remains [ia a], i.e., "ia" - inactive or "a" - active [ia a]. Note that these values are from one iteration of the 2-holdout experiment 


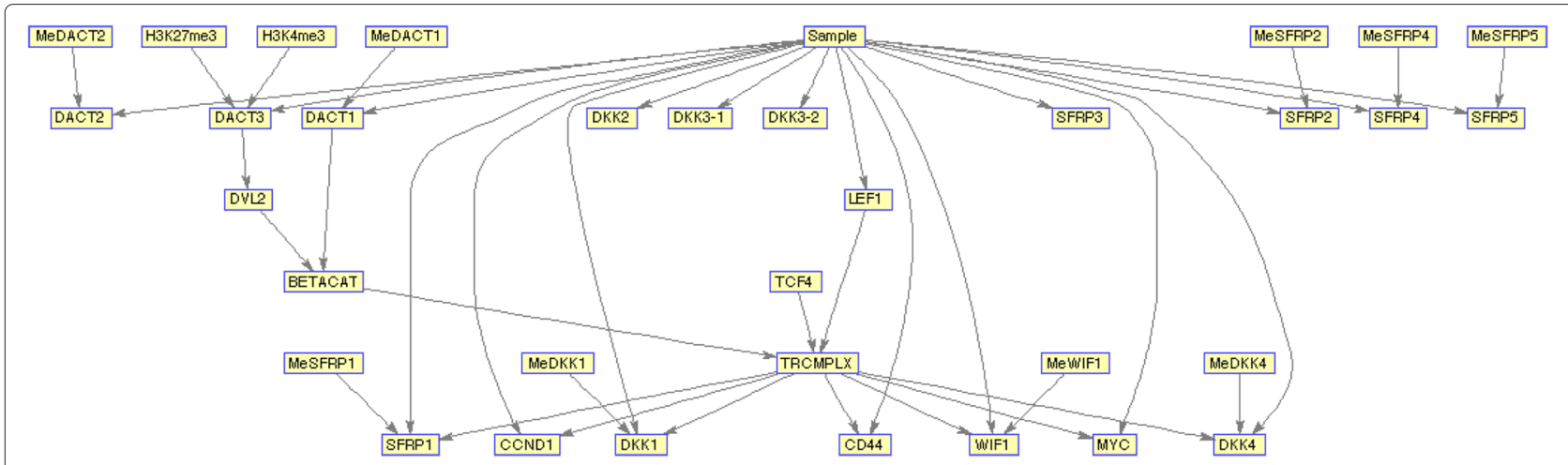

Fig. 2 Influence diagram of $\mathcal{M}_{\mathrm{PBK}+\mathrm{El}}$ contains partial prior biological knowledge and epigenetic information in the form of methylation and histone modification. In this model, the state of Sample is distinguished from state of TRCMPLX that constitutes the Wnt pathway

prototype model. Also, this dataset though not complete, contains enough information to design small computational experiments to test certain biological hypothesis which will be seen later.

From one point of view, this paper's analysis is essentially an exercise in biomarker validation: do the genes selected for follow-up predict tumor status of tissue samples? In the implementation used here, they do not do so with full reliability. This raises the question of the validity of using the small subset of the WNT pathway chosen as a predictive biomarker of tumor statusThis is true! That is why the idea was to segregate the node Sample from TRCMPLX and check the biological hypothesis whether the active (inactive) state of transcription complex is directly related to the sample being tumorous (normal), from a computational perspective. It was found that it is not necessary that TRCMPLX is switched on (off) when the sample is tumorous (normal) given a certain gene expression. By developing a biologically inspired model on this small dataset, one is able to detect if the predictions always point to the biological phenomena or not. In this case, the sample being tumorous or normal given the gene expression evidence is based on a Naive Bayes model (similar to [3]) which does not incorporate prior biological knowledge. It is not the small dataset always that matters but how the network is designed that matters. The status of a sample being tumorous/normal might be inferred in a better way if the prior biological knowledge regarding the pathway was also incorporated and the dominant factor like the activation of transcription complex along with established biomarkers was studied. Sinha [1] gave an improvement over the model implemented in [3] for this very reason.
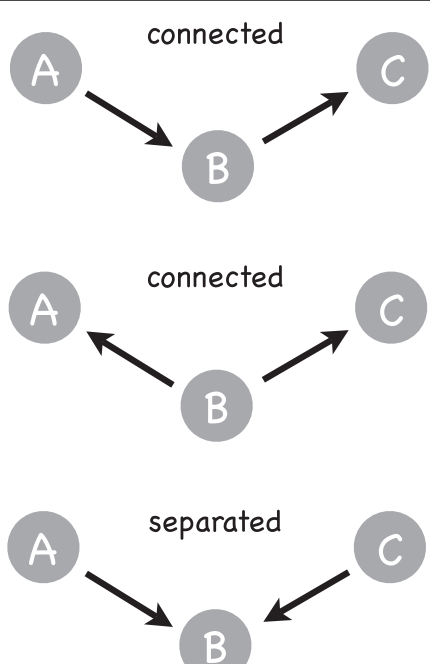

I.

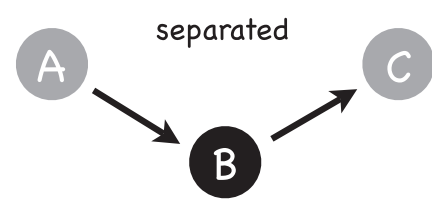

II.

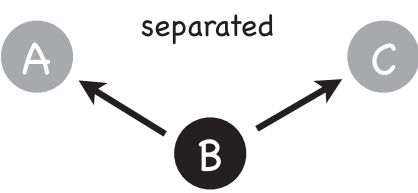

III.

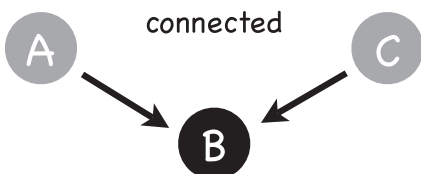

Fig. 3 Cases for d-connectivity and d-separation. Black (gray) circles mean that evidence is available (not available) regarding a particular node 


\subsection{Design of experiments}

A two holdout experiment is conducted in order to reduce the bias induced by unbalanced training data. From a machine learning perspective, this bias is removed by selecting one sample from normal and one sample from tumor for testing purpose and the remaining samples to form the training dataset. The procedure of selection is repeated for all possible combinations of a normal sample and a tumor sample. What happens is that the training data remains balanced and each pair of test sample (one normal and one tumor) gets evaluated for prediction of the label. Repetitions of a normal (tumor) sample across test pairs give equal chance for each of the tumor (normal) sample to be matched and tested.

\subsection{Inference and statistical tests}

The inference of the biological relations is done by feeding the the evidence into the model and computing the conditional probability of the effect of a factor(s) given the evidence. Note that the Bayesian network used in the BNT toolbox by [4] uses the two-pass junction tree algorithm. In the first pass, the Bayesian network engine is created and initialized with prior and estimated probabilities for the nodes in the network. In the second pass, after feeding in the evidence for some of the nodes, the parameters for the network are recomputed. It is these recomputed parameters that give insight into the hidden biological relations based on the design of the network as well as the use of the principle of $d$ connectivity/separability. Since the computed conditional probabilities may change depending on the quality of evidence per test sample that is fed to the network, statistical estimates are deduced and receiver operator curves (ROC) along with respective their area under the curve (AUC) are plotted. These estimates give a glimpse of the quality of predictions. Apart from this, since a distribution of predictions is generated via 2-holdout experiment, Kolmogorov-Smirnov test is employed to check the statistical significance between the distributions. The significance test helps in comparing the prediction results for hypothesis testing in different models and thus point to the effectiveness of the models regarding biological interpretations.

This non-parametric test will reject the null hypothesis when distributions differ in shape. The author notes that his more complex biologically inspired models give significant KS test $p$ values when comparing predictions of the $\beta$-catenin transcription factor complex state and the tumor/non-tumor status of the samples. While the result is interesting, the KS test adds little information on interpretation. Are the biological models incorrect? Are the predictions produced using faulty assumptions? Are false positives or false negatives more frequent, and if so why?
Biological models might be lacking in biological information and correctness depends on how the model is designed. This does not mean that the inferences are wrong and the assumptions are faulty. The differences in the distribution is due to the prior biological knowledge that has been incorporated into the models. So indirectly, the KS test points to the significance of adding the biological data. While using the naive Bayes model (from [3]), it was found that the prediction accuracy was almost $100 \%$. But w.r.t. issue raised regarding the biomarker prediction earlier, the accuracy value drops due to the model complexity and correct biological inferences can be made. From the Bayesian perspective, the numerical value represents a degree of belief in an event and the $100 \%$ prediction accuracy might not capture the biological phenomena as well as the influence of the biomarker properly from the naive Bayes model with minimal prior biological knowledge in [3] and [1]. Thus, KS test gives an indirect indication regarding the significance of using the prior biological knowledge in comparison to the negligible knowledge while designing the models.

\subsection{MATLAB and Bayesian network toolbox}

The choice of MATLAB was made purely because of its ability to handle various types of data structures which can be used for fast prototype building. Also, the BNT toolbox is freely available and provides most of the functions necessary to deal with the design of the Bayesian network models of different types (both static and dynamic). There are many packages freely available in $R$ that could be used for development of these projects, but they lack the level of details that the BNT toolbox provides. The downside of the BNT toolbox is that one needs a MATLAB license. Finally, the BNT toolbox can be downloaded from https://code.google.com/p/bnt/. Instructions for installations as well as how to use the package is available in the website. The material from [1] has been made available in the Google drive https://drive.google.com/ folderview?id=0B7Kkv8wlhPU-T05wTTNodWNydjA\& usp=sharing. This contains the individual files, contents of which are used in this manuscript. The drive and its contents can be accessed via the URLs mentioned earlier in the abstract. To ease the understanding of the knowhow-it-works of BNT toolbox, the drive contains two files namely sprinkler_rain_script.m and sprinkler_rain.mat. The former contains code from BNT toolbox in a procedural manner and the latter contains the saved results after running the script. As a toy example, these can be used for quick understanding.

An important point of observation-while executing the code-if the chunks of code are not easy to follow, then please use the MATLAB facility of debugging by setting up breakpoints and a range of functions starting with prefix 
DB. Note that the breakpoints appear as solid red dots on the left hand side of the MATLAB editor when being used. When the code is running, solid green arrows stop at these breakpoints and let the user analyze the query of interest. More help is available on Internet as well as via the MATLAB help command.

\section{Modeling and simulation}

\subsection{Data collection and estimation}

An important component of this project is the Bayesian network toolbox provided by [4] and made freely available for download on https://code.google.com/p/bnt/ as well as a MATLAB license. Instructions for installations are provided on the mentioned website. To begin the project, one can make a directory titled temp with a subdirectory named data and transfer the geneExpression.mat file into data.

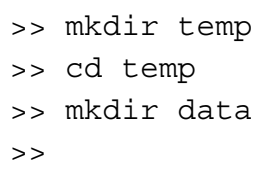

This .mat file contains expression profiles from [2] for genes that play a role in Wnt signaling pathway at an intra/extracellular level and are known to have inhibitory effect on the Wnt pathway due to epigenetic factors. For each of the 24 normal mucosa and 24 human colorectal tumor cases, gene expression values were recorded for 14 genes belonging to the family of SFRP, DKK, WIF1, and $D A C T$. Also, expression values of established Wnt pathway target genes like $L E F 1, M Y C, C D 44$, and CCND1 were recorded per sample.

The directory temp also contains some of the .m files, parts of the contents of which will be explained in the order of execution of the project. The main code begins with a script titled twoHoldOutExp.m (Note that the original unrefined file is under the name twoHoldOutExporiginal.m). This script contains the function twoHoldOutExp which takes two arguments named eviDence and model. eviDence implies the evidence regarding "ge" for gene evidence, "me" for methylation, "ge+me" for both gene and methylation, while model implies the network model that will be used for simulation. Sinha [1] uses three different models, i.e., "t1" or $\mathcal{M}_{\mathrm{PBK}+\mathrm{EI}}$ that contains prior biological knowledge as well as epigenetic information, "t2" or $\mathcal{M}_{\mathrm{PBK}}$ that contains only prior biological knowledge, and, finally, "p1" or $\mathcal{M}_{\mathrm{NB}+\mathrm{MPBK}}$ that is a modified version of the naive Bayes framework from [3]. On the MATLAB command prompt, one can type the following

$>$ twoHoldOutExp ('ge', 't $\left.t 1^{\prime}\right)$
The code begins with the extraction of data from the gene expression matrix by reading the geneExpression.mat file via the function readCustomFile in the readCustomFile. $m$ and generates the following variables as the output: (1) uniqueGenes-name of genes gleaned from the file, (2) expressionMatrix-2D matrix containing the gene expression per sample data, (3) noGenes-total number of genes available, (4) nosamples-total number of samples available, (5) groundTruthLabels-original labels available from the files, and (6) transGroundTruthLabels-labels transformed into numerals.

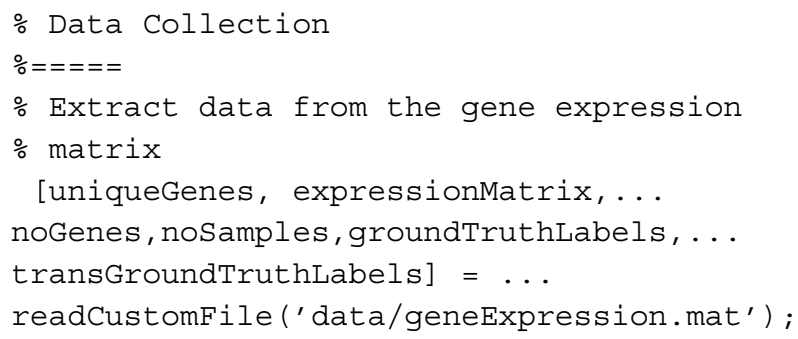

\subsection{Assumed and estimated probabilities from literature} Next, the probability values for some of the nodes in the network is loaded depending on the type of the network. Why these assumed and estimated probabilities have been addressed in the beginning of the computation experiment is as follows. It can be seen that the extra/intracellular factors affecting the Wnt pathway in the dataset provided by [2] contain some genes whose expression is influenced by epigenetic factors mentioned in Table 3. Hence, it is important to tabulate and store prior probability values for known epigenetic biological factors that influence the pathway. Other than the priors for epigenetic nodes, priors for some of the nodes that are a major component of the pathway but do not have data from prior approximation, are assumed based on expert knowledge. Once estimated or assumed based on biological knowledge, these probabilities need not be recomputed and are thus stored in proper format at the beginning of the computational experiment.

The estimation of prior probabilities is achieved through the function called dataStorage in the file dataStorage. $m$. The function takes the name of the model as an input argument and returns the name of the file called probabilities.mat in the variable $\mathrm{f} i \mathrm{lename}$. The .mat file contains all the assumed and computed probabilities of nodes for which data is available and is loaded into the workspace of the MATLAB for further use. The workspace is an area which stores all the current variables with their assigned instances such that the variables can be manipulated either interactively via command prompt or from different functions. 
\% Load probability values for some of

$\%$ the nodes in the network

fname = datastorage $($ model $)$;

load (fname);

$\mathcal{M}_{\mathrm{PBK}+\mathrm{EI}}($ model $=$ "t1") requires more prior estimations than $\mathcal{M}_{\mathrm{PBK}}\left(\right.$ model $=$ "t2") and $\mathcal{M}_{\mathrm{NB}}($ model $=\mathrm{p} 1)$, due to use of epigenetic information. Depending on the type of model parameter fed to the function dataStorage, the probabilities for the following factors are estimated:

1. Repressive histone mark H3K27me3 for DACT3 11 loci from [2] was adopted. Via fold enrichment, the effects of the $H 3 K 27 m e 3$ were found $500 \mathrm{bp}$ downstream of and near the DACT3 transcription start site (TSS) in HT29 cells. These marks were recorded via chromatin immuno-precipitation (ChiP) assays and enriched at 11 different loci in the 3.5- to $3.5-\mathrm{kb}$ region of the DACT3 TSS. Fold enrichment measurements of $H 3 K 27 \mathrm{me} 3$ for normal FHs74Int and cancerous SW480 were recorded and normalized. The final probabilities are the average of these normalized values of enrichment measurements.

2. Active histone mark $H 3 K 4 m e 3$ for $D A C T 3$ loci from [2] was adopted. Via fold enrichment, the effects of the H3Kme 3 were found 500 bp downstream of and near the DACT 3 transcription start site (TSS) in HT29 cells. These marks were recorded via chromatin immuno-precipitation (ChiP) assays and enriched at 11 different loci in the 3.5- to 3.5-kb region of the $D A C T 3$ TSS. Fold enrichment measurements of $H 3 \mathrm{~K} 4 \mathrm{me} 3$ for normal FHs74Int and cancerous $S W 480$ were recorded and normalized. The final probabilities are the average of these normalized values of enrichment measurements.

3. Fractions for methylation of $D K K 1$ and WIF1 gene taken from [5] via manual counting through visual inspection of intensity levels from methylationspecific PCR (MSP) analysis of gene promoter region and later normalized. These normalized values form the probability estimates for methylation.

4. Fractions for methylation and non-methylation status of SFRP1, SFRP2, SFRP4, and SFRP5 (CpG islands around the first exons) was recorded from six affected individuals each having both primary CRC tissues and normal colon mucosa from [6] via manual counting through visual inspection of intensity levels from MSP analysis of gene promoter region and later normalized. These normalized values form the probability estimates for methylation.

5. Methylation of $D A C T 1$ ( +52 to +375 BGS) and $D A C T 2$ (+52 to +375 BGS) in promoter region for
Normal, HT29, and RKO cell lines from [2] was recorded via counting through visual inspection of open or closed circles indicating methylation status estimated from bisulfite sequencing analysis and later normalized. The averaged values of these normalizations form the probability estimates for methylation.

6. Concentration of $D V L 2$ decreases with expression of $D A C T 3$ and vice versa [2]. Due to the lack of exact proportions, the probability values were assumed.

7. Concentration of $\beta$-catenin-given concentrations of $D V L 2$ and $D A C T 1$ varies; and for static model, it is tough to assign probability values. High $D V L 2$ concentration or suppression (expression) of $D A C T 1$ leads to increase in the concentration of $\beta$-catenin $[2,7]$. Wet lab experimental evaluations might reveal the factual proportions.

8. Similarly, the concentrations of TRCMPLX $[8,9]$ and TCF4 [3] have been assumed based on their known roles in the Wnt pathway. Actual proportions as probabilities require further wet lab tests.

9. Finally, the probability of Sample being tumorous or normal is a $50 \%$ chance level as it contains an equal amount of cancerous and normal cases.

Note that all these probabilities have been recorded in Table 1 of [1] and their values stored in the probabilities.mat file.

\subsection{Building the Bayesian network model}

Next comes the topology of the network using prior biological knowledge which is made available from the results of wet lab experiments documented in literature. This network topology is achieved using the function generatelnteraction in the file generateInteraction.m. The function takes in the set of uniqueGenes and the type of the model and generates a cell of interaction for the Bayesian network as well as a cell of unique set of names of the nodes, i.e., Nodenames. A cell is like a matrix but with elements that might be of different types. The indexing of a cell is similar to that of a matrix except for the use of parenthesis instead of square brackets. interaction contains all the prior established biological knowledge that carries causal semantics in the form of arcs between the parent and child nodes. It should be noted that even though the model is not complete due to its static nature, it has the ability to encode prior causal relationships and has the potential for further refinement. Note that a model not being complete does not conclude that the results will be wrong.

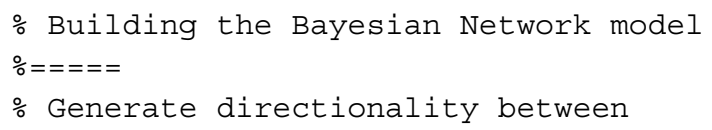




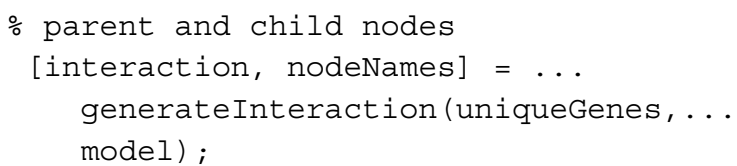

The interaction and nodeNames are used as input arguments to the function mk_adj_mat, which then generates an adjacency matrix for a directed acyclic graph (DAG) stored in dag. Using functions biograph and input arguments dag and nodeNames generates a structure gObj that can be used to view the topology of the network. A crude representation of $\mathcal{M}_{\mathrm{PBK}+\mathrm{EI}}$ and $\mathcal{M}_{\mathrm{NB}+\mathrm{MPBK}}$ shown in Figs. 2 and 4 was generated using the function view.

\% Generate dag for the interaction

\% between nodeNames

dag = mk_adj_mat (interaction, nodeNames, 0);

\% To visualise the graphs or bayesian

\% network

gobj = biograph (dag, nodeNames)

gObj = view $(g O b j)$;

Once the adjacency matrix is ready, the initialization of the Bayesian network can be done easily. The total number of nodes is stored in $\mathrm{N}$ and the size of the nodes are defined in nodesizes. In this project, each node has a size of two as they contain discrete values representing binary states. Here, the function ones defines a row vector with $\mathrm{N}$ columns. The total number of discrete nodes is defined in discreteNodes. Finally, the Bayesian network is created using the function mk_bnet from the BNT that takes the following as input arguments: (1) dag-the adjacency matrix, (2) nodesizes-defines the size of the nodes, and (3) discreteNodes-the vector of nodes with their indices marked to be discrete in the Bayesian network and dumps the network in the variable bnet. bnet is of the type STRUCTURE which contains fields, each of which can be of different types like vector, character, array, matrix, cell, or structure. The contents of a field of a structure variable (say bnet), with proper indices, if necessary can be accessed and seen using "bnet.fieldname."

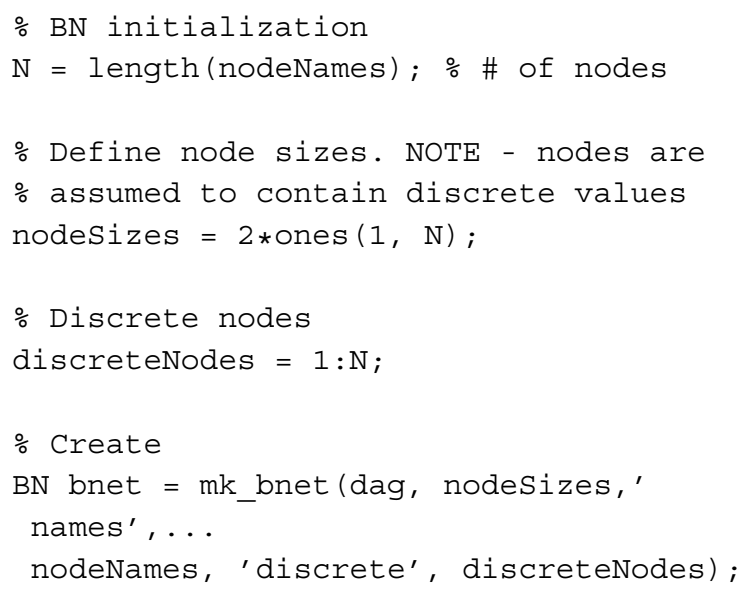

\subsection{Holdout experiment}

After the framework of the Bayesian network has been constructed and initialized, the holdout experiment is conducted. The purpose of conducting the experiment is to generate results on different test data while training the Bayesian network with different sets of training data. From [1], the design of the experiment is a simple 2-holdout experiment where one sample from the normal and one sample from the tumor are paired to form a test dataset. Excluding the pair formed in an iteration of 2-holdout experiment, the remaining samples are considered for training of a $\mathrm{BN}$ model. Thus, in a dataset of 24 normal and 24 tumorous cases, an iteration will have a training set which will contain 46 samples and a test set which will contain 2 samples (one of normal and one of tumor). This procedure is repeated for every normal sample which is combined with each of the tumorous sample to form a series of test dataset. In total, there will be 576 pairs of test data and 576 instances of training data. Note that for each test sample in a pair, the expression value for a gene is discretized using a threshold computed for that particular gene from the training set. Computation of threshold will be elucidated later. This computation is repeated for all genes per test sample. Based on the available evidences from the state of expression of all genes that constitute the test data, inference regarding the state of the both $\beta$-catenin transcription complex and the test sample is made. These inferences reveal (a) hidden biological relationship between the expressions of the set of genes under

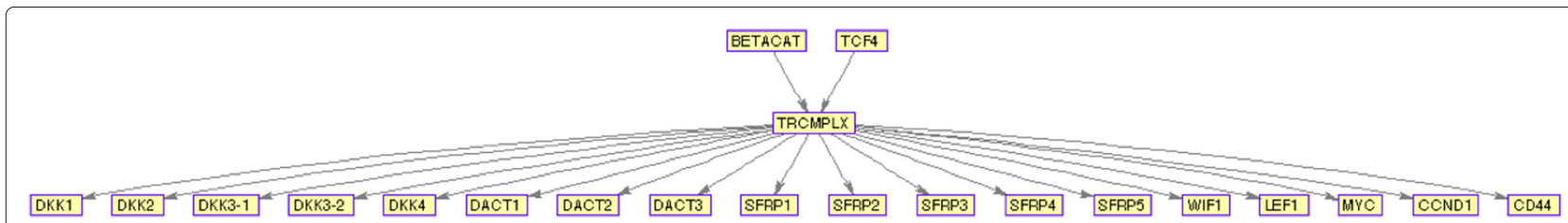

Fig. 4 Influence diagram of $\mathcal{M}_{\mathrm{NB}+\mathrm{MPBK}}$ is a naive Bayes model that contains minimal prior biological knowledge. In this model, the state of TRCMPLX is assumed to be indicate whether the sample is cancerous or not 
consideration and the $\beta$-catenin transcription complex and (b) information regarding the activation state of the $\beta$-catenin transcription complex and the state of the test sample, as a penultimate step to the proposed hypothesis testing. Two-sample Kolmogorov-Smirnov (KS) test was employed to measure the statistical significance of the distribution of predictions of the states of the previously mentioned two factors.

Apart from testing the statistical significance between the states of factors, it was found that the prediction results for the factors obtained from models including and excluding epigenetic information were also significantly different. The receiver operator curve (ROC) graphs and their respective area under the curve (AUC) values indicate how the predictions on the test data behaved under different models. Ideally, high values of AUC and steepness in ROC curve indicate good quality results.

The holdout experiment begins with the computation of the total number of positive and negative labels present in the whole dataset as well as the search of the indices of the labels. For this, the values in the variable noSamples and transGroundTruthLabels computed from function readCustomfile are used. noPos (noNeg) and posLabelIdx (negLabelIdx) store the number of positive (negative) labels and their indices, respectively.

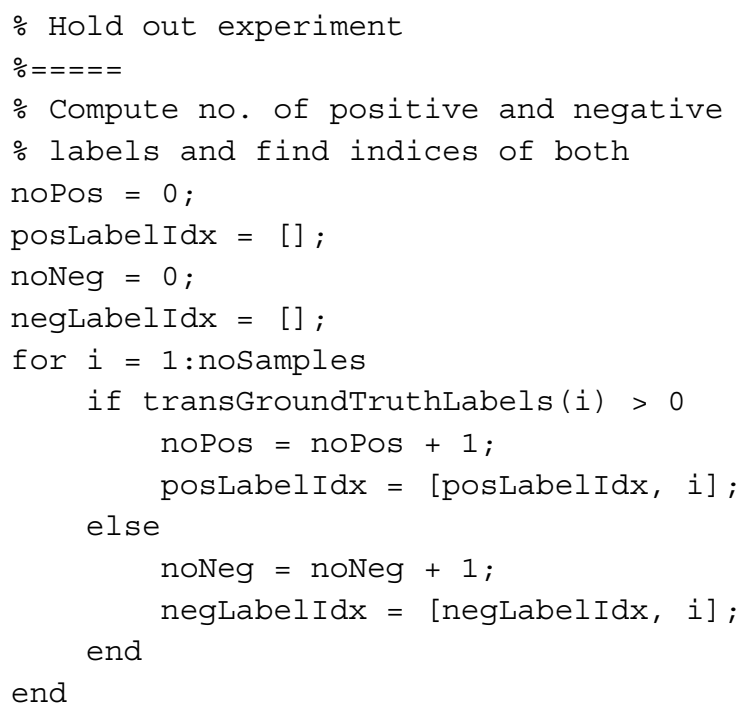

For storing results as well as the number of times the experiment will run, variables runCnt and Runs are initialized. Runs is of the type structure. The condition in the if statement is not useful now and will be described later.

runCnt $=0$;

Runs $=\operatorname{struct}([])$;

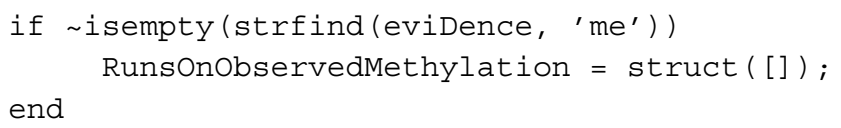

For each and every positive (cancerous) and negative (normal) labels, the number of times the experiments run is incremented in the count variable runCnt. Next, the indices for test data is separated by using the $i$ th positive and the $j$ th negative label and these indices are stored in testDataIdx. The test data itself is then separated from expressionMatrix using the testDataIdx and stored in dataForTesting. The corresponding ground truth labels of the test data are extracted from transGroundTruthLabels using testDataIdx and stored in labelForTesting.

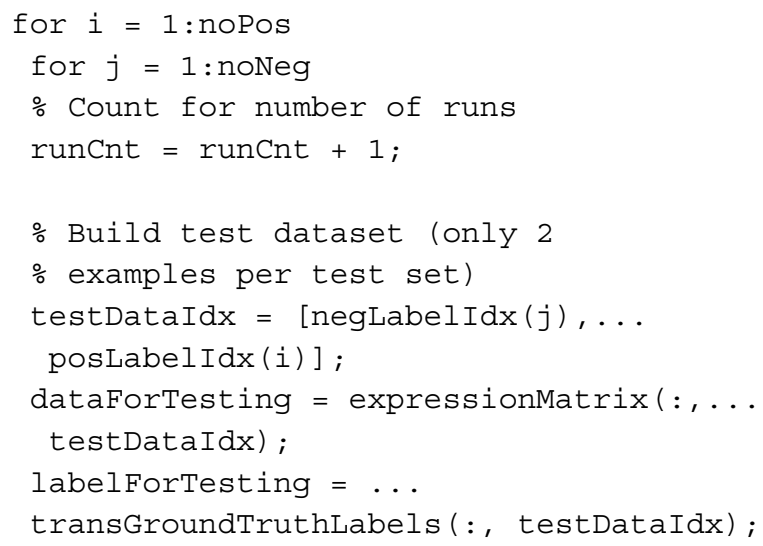

After the storage of the test data and its respective indices, trainingDataIdx is used to store the indices of training data by eliminating the indices of the test data. This is done using temporary variables tmpPosLabelIdx and tmpNegLabelIdx. trainingDataIdx is used to store the training data in variable dataForTraining using expressionMatrix and the indices of training data in variable labelforTraining using transGroundTruthLabels.

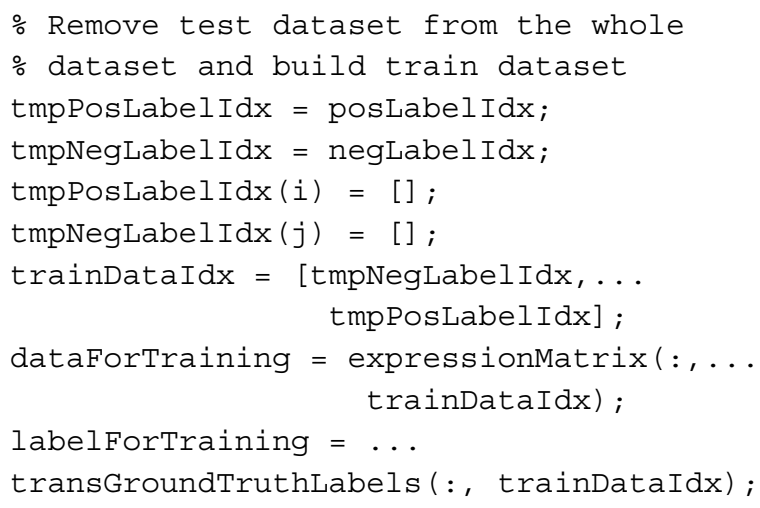




\subsubsection{Defining and estimating probabilities and conditional probabilities tables for nodes in bnet}

Till now, the probabilities as well as conditional probability tables (cpt) for some of the nodes have been stored in the probabilities.mat file and loaded in the workspace. But the cpt for all the nodes in the bnet remain uninitialized. The next procedure is to initialize the tables using assumed values for some of the known nodes while estimating the entries of cpt for other nodes (i.e., of nodes representing genes) using the training data.

To this end, it is important to define a variable by the name cpdStorage of the format structure. Starting with all the nodes that have no parents and whose probabilities and cpt have been loaded in the workspace (saved in probabilities.mat), the for loop iterates through all the nodes in the network defined by $\mathrm{N}$, stores the index of the $k$ th node in nodeidx using function bnet.names with input argument nodeNames $\{\mathrm{k}\}$ and assigns values to cpt depending on the type of the model. If $\mathcal{M}_{\mathrm{PBK}+\mathrm{EI}}$ (model = "t1") is used and the $k$ th entry in nodeNames matches with TCF4, then the cpt value in PrTCF4 is assigned to cpt. The parent node of this node is assigned a value 0 and stored in cpdStorage $(\mathrm{k})$. parentnode $\{1\}$. The name TCF4 or nodeNames $\{\mathrm{k}\}$ is assigned to cpdStorage $(\mathrm{k})$.node. The cpt values in cpt is assigned to cpdStorage $(\mathrm{k})$. cpt. Finally, the conditional probability density cpt for the node with name TCF4 is stored in bnet.CPD using function tabular_CPD, the Bayesian network bnet, the node index nodeidx, and cpt. Similarly, values in PrMeDKK1, avgPrMeDACT1, avgPrMeDACT2, avgPrH3K27me3, avgPrH3K4me3, PrMeSFRP1, PrMeSFRP2, PrMeSFRP4, PrMesfRP5, PrMeWIF1, and PrSample initialize the cpt values for nodes $M e D A C T 1, M e D A C T 2$, H3k27me3, H3k4me3, MeSFRP1, MeSFRP2, MeSFRP4, MeSFRP5, MeWIF1, and Sample, respectively. It might not be necessary to hard code the variables and more efficient code could be written. Currently, the selection of the hard-coded variables is for ease in reading the code from a biological point of view for person with computer science background. But surely, this programming style is bound to change when large and diverse datasets are employed.

Similar initializations happen for models $\mathcal{M}_{\mathrm{PBK}}$ (model $=$ "t2") and $\mathcal{M}_{\mathrm{NB}+\mathrm{MPBK}}($ model $=$ "p1"). It should be noted that in $\mathcal{M}_{\mathrm{PBK}}\left(\mathcal{M}_{\mathrm{NB}+\mathrm{MPBK}}\right)$, the only nodes without parents are TCF4 and Sample (TCF4 and BETA$C A T)$. To accommodate for these models, the necessary elseif statements have been embedded in the for loop below.

- Define P and CPD for the nodes of the

$\%$ bnet

cpdstorage $=$ struct $([])$;

\% Store probabilities for nodes with no

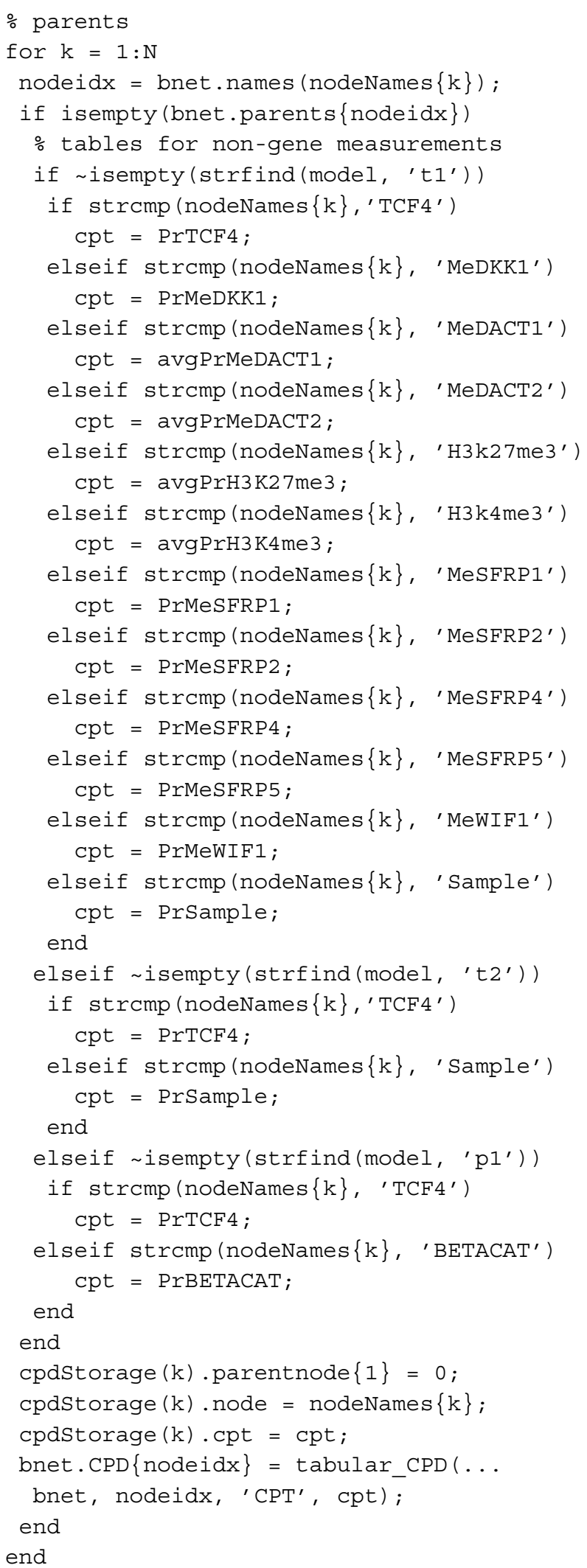

In the same for loop above, the next step is to initialize probability as well as the cpt values for nodes 
with parents. Two cases exist in the current scenario, i.e., nodes that (1) represent genes and (2) do not represent genes. To accommodate for gene/non-gene node classification, a logical variable GENE is introduced. Also, before entering the second for loop described below, a variable gene_cpd of the format structure is defined for storage of the to be computed cpt values for all genes in the dataset. parentidx stores the indices of the parents of the child node under consideration using the child's index in nodeidx via bnet. parents $\{$ nodeidx $\}$. The total number of parents a child node has is contained in noParents.

Initially, GENE is assigned a value of 0 indicating that the node under consideration is not a gene node. If this is the case, the GENE in the if condition of the for loop below gets executed. In this case, depending on the type of the model cpt values of a particular node is initialized. For $\mathcal{M}_{\mathrm{PBK}+\mathrm{EI}}$ and $\mathcal{M}_{\mathrm{PBK}}$ (model $=$ "t1" and model = "t2"), the cpt values for nodes BETA$C A T, D V L 2$, and TRCMPLX is stored using values in PrBETACAT, PrDVL2, and PrTRCMPLX. As before, using the function tabular_CPD and values in nodeidx, bnet, and cpt as input arguments, the respective cpt is initialized in bnet. $\operatorname{CPD}\{$ nodeidx $\}$. Similar computations are done for $\mathcal{M}_{\mathrm{NB}+\mathrm{PBK}}$, i.e., model "p1" for node TRCMPLX. Finally, the indices of the parents of the $k$ th child node are stored in cpdStorage $(\mathrm{k})$ - parentnode $\{\mathrm{m}\}$.

On the other hand, if the name of the node in the $k$ th index of nodeNames matches the name in the $l$ th index of uniqueGenes, a parent variable of format cell is defined within the second nested for loop below. The names of the parents are stored in this variable using nodeNames $\{\operatorname{parentidx}(\mathrm{n})\}$. Next, the cpt values of these parent nodes are separately stored using a cell parent_cpd and a count cnt. Finally, the cpd values for the $l$ th gene is determined using the function generateGenecpd in the script generateGenecpd.m that takes the following input arguments: (1) vecTraining-gene expression from training data, (2) labelTraining-labels for training data, (3) nodeName-name of the gene involved, (4) parent-name of parents of the child node or the gene under consideration, (5) parent_cpd-parent cpd values, (6) model-kind of model and finally returns the output as a structure gene_cpd containing cpd for the particular gene under consideration given its parents as well as a threshold value in the form of median. In the code below, the values of the following variables are used as input arguments for the function generateGenecpd, in order: (1) dataForTraining $(1,:)$-training data for the $l$ th unique gene, (2) labelForTraining-labels for the training data, (3) uniqueGenes $\{1\}$, (4) parent, (5) parent_cpd, (6) model. The output of the function is stored in the structure variable $\mathrm{x}$. The threshold at which the probabilities were computed for the $l$ th gene is stored in gene_cpd( 1 ).vecmedian using $\mathrm{x}$. vecmedian and the probabilities themselves are stored in gene_cpd ( 1 ). T using $\mathrm{x}$. T. These probabilities are reshaped into a row vector and stored in cpt. As mentioned before, using function tabular_CPD and values in nodeidx, bnet and cpt as input arguments, the respective cpt is initialized in bnet. $\operatorname{CPD}\{$ nodeidx $\}$. Finally, the required values of cpt, name of $l$ th gene or $k$ th node and indices of its parent nodes are stored in cpdStorage (k).cpt, cpdStorage (k). node and cpdStorage $(k)$. parentnode $\{m\}$, respectively.

It should be noted that the exposition of the generation of probability values for the different genes via the function generateGenecpd needs a separate treatment and will be addressed later. To maintain the continuity of the workflow of the program, the next step is addressed after the code below.

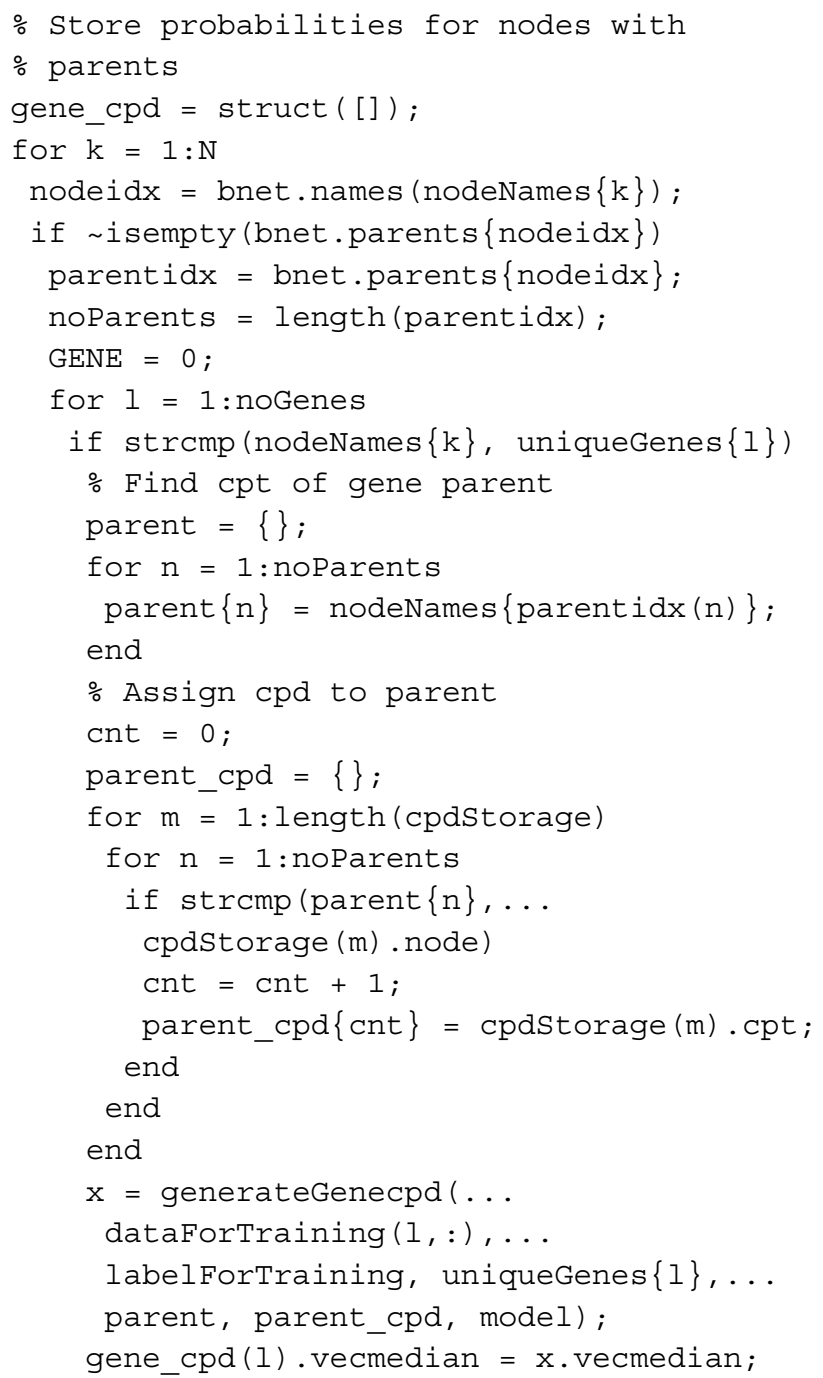




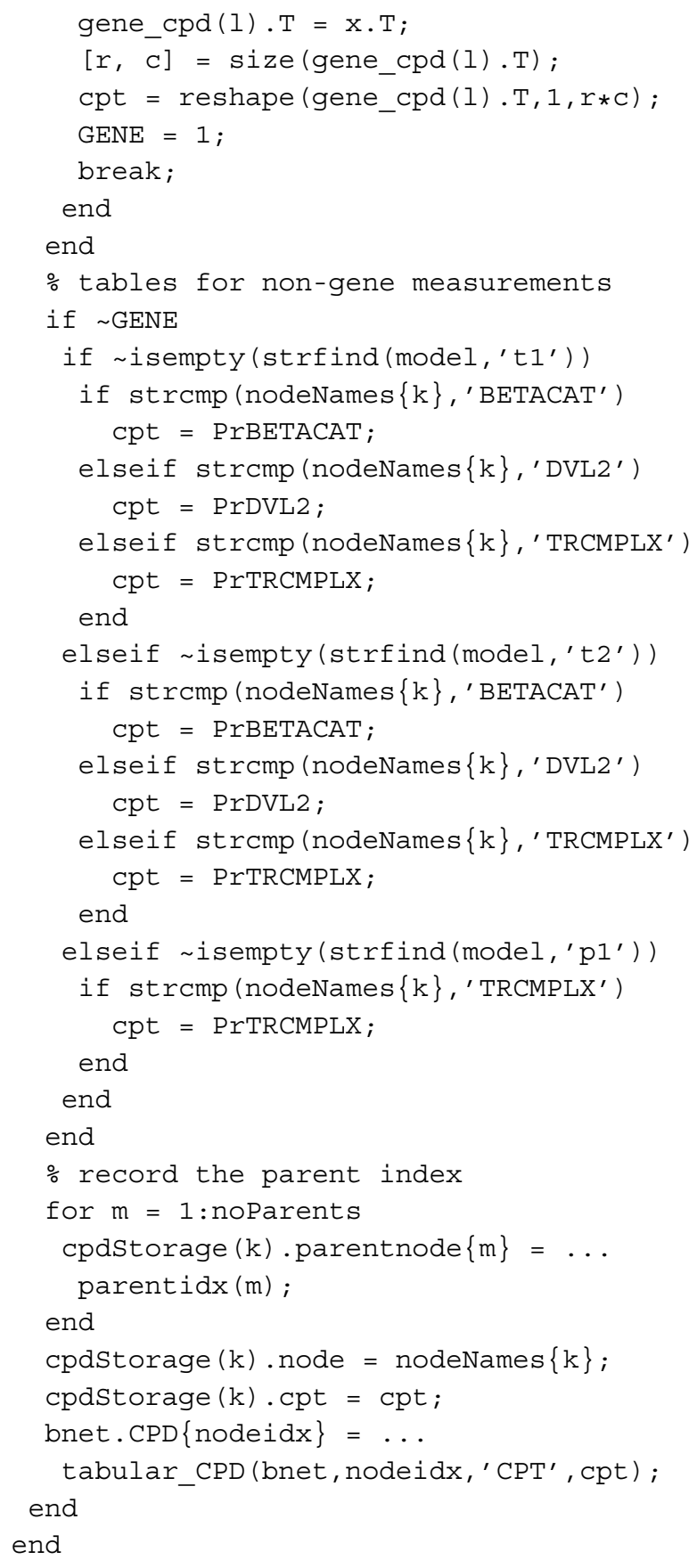

\subsubsection{Evidence building and inference}

The values estimated in gene_cpd as well as cpdStorage are stored for each and every run of the holdout experiment. Also, the dimensions of the testing data are stored.

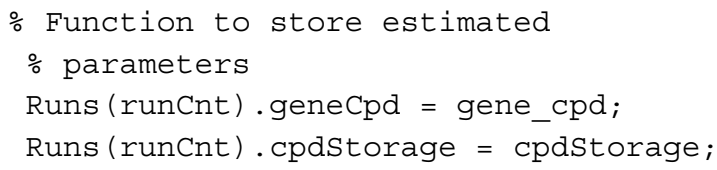

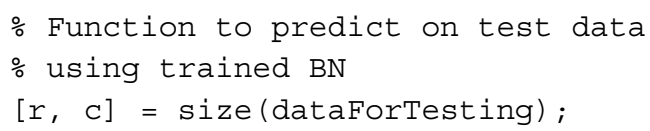

Next, depending on the type of the evidence provided in eviDence, inferences can be made. Below, a section of code for the gene expression evidence, which gets executed when the case "ge" matches with the parameter eviDence of the switch command, is explained. The issue that was to be investigated was whether the $\beta$-catenin-based TRCMPLX is always switched on (off) or not when the Sample is cancerous (normal). In order to analyze this biological issue from a computational perspective, it would be necessary to observe the behavior of the predicted states of both TRCMPLX as well as Sample, given all the available evidence. For this purpose, the variable tempTRCMPLXgivenAllge is defined as a vector for each model separately, while the variable temPSAMPLE is defined as a vector for biologically inspired models, i.e., $\mathcal{M}_{\mathrm{PBK}+\mathrm{EI}}$ and $\mathcal{M}_{\mathrm{PBK}}$ separately. This is due to the assumption that the state of TRCMPLX is the same as the state of the test sample under consideration in the $\mathcal{M}_{\mathrm{NB}+\mathrm{MPBK}}$ (a modification of [3]).

In the section of the code below, for each of the test dataset, an evidence variable of the format cell is defined. The evidence is of the size equivalent to the number of node $\mathrm{N}$ in the network. Only those indices in the cell will be filled for which information is available from the test data. Since the function twoHoldOutExp started with "ge" as an argument for the type of evidence, evidence will be constructed from information available via gene expression from the test data. Thus for the $m$ th gene, if the gene expression in the test data (i.e., dataForTesting $(\mathrm{m}, \mathrm{k})$ ) is lower than the threshold generated using the median of expressions for this gene in the training data (i.e., gene cpd (m).vecmedian), then the evidence for this gene is considered as inactive or repressed, i.e., evidence $\{$ bnet. names (uniqueGenes (m)) $\}=1$, else the evidence for this gene is considered as active or expressed, i.e., evidence\{bnet.names (uniqueGenes $(\mathrm{m}))\}=2$. Iterating through all the genes, the evidence is initialized with the available information for the $k$ th test data.

Once the probability values have been initialized either by computation or assumption, then for the $k$ th test data, a Bayesian network engine is generated and stored in bnetEngine via the junction tree algorithm implemented in function jtree inf engine that uses the input argument as the newly initialized network stored in bnet. The bnetEngine is then fed with the values in evidence to generate a new engine that contains the updated probability values for nodes for which there is no evidence in the network. This is done using the function 
enter_evidence. According to BNT provided by [4], in the case of the jtree engine, enter_evidence implements a two-pass message-passing scheme. The first return argument (engine) contains the modified engine, which incorporates the evidence. The second return argument (loglik) contains the log-likelihood of the evidence. It is the first returned argument or the modified engine that will be of use further. It is important to note that for every iteration that points to a new test data in the for loop, a new Bayesian network engine is generated and stored in bnetEngine. If this is not done, then the phenomena of explaining away can occur on feeding new evidence to an already modified engine which incorporated the evidence from the previous test data. In explaining away, the entering of new evidence might outweigh the effect of an existing influencing factor or evidence thus making the old evidence redundant. This simulation is not related to such study of explaining away.

The belief that the TRCMPLX is switched on given the gene expression evidence, i.e., $\operatorname{Pr}(T R C M P L X=2 \mid$ ge as evidence) is computed by estimating the marginal probability values using the function marginal_nodes which takes the engine stored in engine and the name of the node using bnet. names ('TRCMPLX'). The marginal probabilities are stored in margTRCMPLX. The final probability of TRCMPLX being switched on given all gene expression evidences is stored in tempTRCMPLX givenAl lge using margTRCMPLX. T (2). Similarly, for biologically inspired models the belief that the test Sample is cancerous given the gene expression evidence, i.e., $\operatorname{Pr}$ (Sample $=2 \mid$ ge as evidence) is computed using function marginal_nodes that takes the engine stored in engine and the name of the node using bnet. names ('Sample'). The marginal probabilities are stored in margSAMPLE. The final probability of Sample being cancerous given all gene expression evidences is stored in tempSAMPLE using margSAMPLE. T (2) .

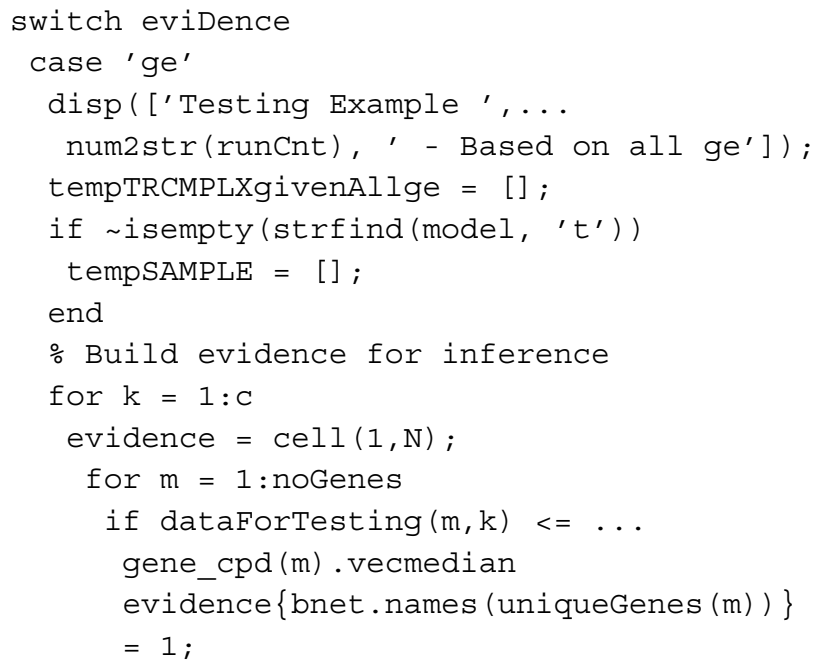

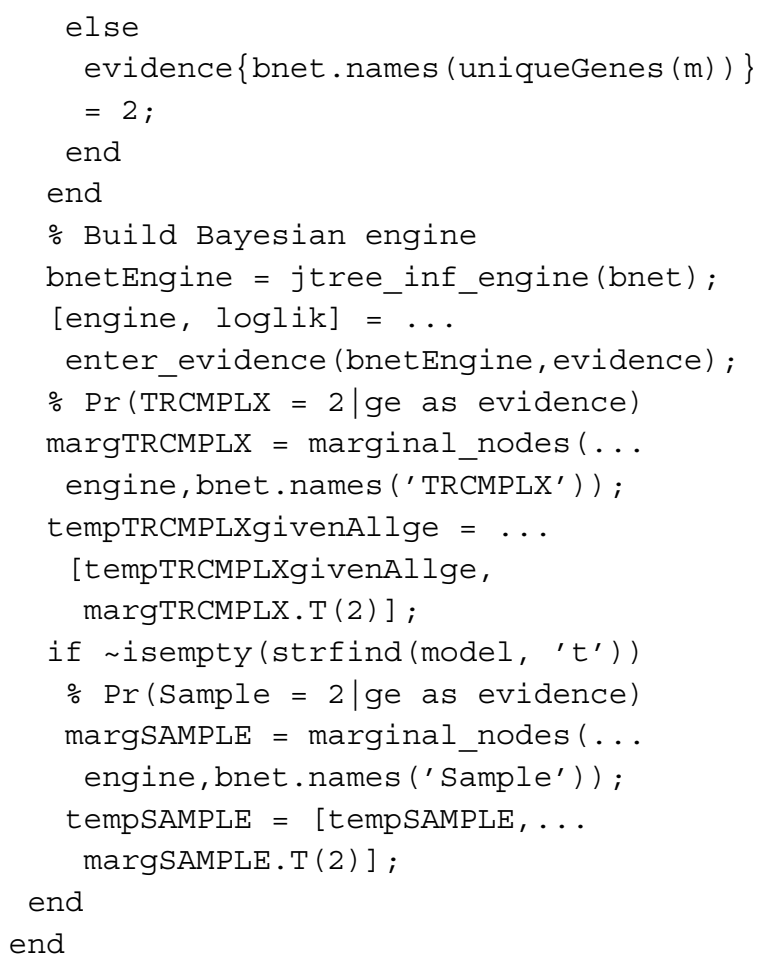

Finally, for a particular count of the run of the experiment, tempTRCMPLXgivenAllge and tempSAMPLE are stored in the structure Runs using different variables associated with Runs. This iteration keeps happening until the 2-holdout experiment is exhausted. The case when eviDence is "me" or evidence for methylation will be discussed later as a programming project.

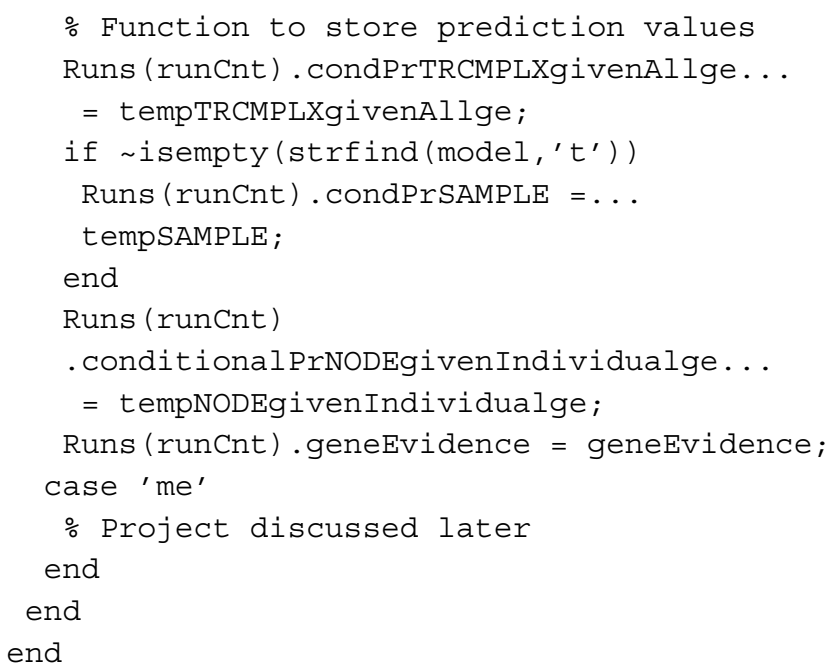

\subsection{Storing results, plotting graphs, and saving files}

The final section of the code deals with the storing of the results, plotting of graphs, and saving the results in the files. Since the current explanation is for gene expression evidence, the code pertaining to "ge" is explained. Readers 
might want to develop the code for evidence regarding methylation as a programming project.

To store results as well as the conditional probabilities for TRCMPLX and Sample given all the gene expression evidence, a cell variable Results, a counter cntResult, and vector variables CondPrTRCMPLXgivenAllge, condPrSAMPLE, and labels are defined as well as initialized. Next, the prediction values and original labels are stored while iterating through the total number of runs of the experiment. This is done using the for loop and the variable runCnt. For the $i$ th run, predicted conditional probabilities of TRCMPLX and Sample from each run are stored in CondPrTRCMPLXgivenAllge $(i,:)$ and condPrSAMPLE $(i,:)$, depending on the model used. Finally, the ground truth labels of the test data are stored in a matrix where the $i$ th row is initialized with labels $(i,:)=[-1,+1] ;$. Here, labels in a matrix and $-1(+1)$ represent normal (cancerous) cases. Next, the variables condPrTRCMPLXgivenAllge and CondPrSAMPLE are reshaped into vectors for further processing.

The plotting of the ROC curves and the estimation of their respective AUCs is achieved using function perfcurve that takes labels and either of the vectors CondPrTRCMPLXgivenAlige or CondPrSAMPLE depending on the type of the model selected. The function churns out useful information in the form of the false positive rate in $\mathrm{X}$, the true positive rate in $\mathrm{Y}$, and the estimated AUC for ROC of CondPrTRCMPLXgivenAlige (COndPrSAMPLE) in AUCTRCMPLXgivenAllge (AUCSAMPLE). The plot function is used to draw the graphs along with the depiction of legends using function legend. Finally, the two-sample Kolmogorov-Smirnov test between the predictions of states of TRCMPLX and Sample is performed using the kstest 2 function. This function takes the two vectors condPrTRCMPLXgivenAllge and CondPrSAMPLE as arguments, compares the distribution of the predictions, and returns the state of significance between the two distributions in h01. If the value of h01 is 1 , then statistical significance exists else it does not exist. Sinha [1] shows that the statistical difference exists between predictions of TRCMPLX and Sample when the nodes for the same are segregated in the biologically inspired causal models, which is not the case with the Naive Bayes model.

Lastly, the computed variables are stored in a .mat file using the function save. Options for using the save function can be obtained from the help command in MATLAB.

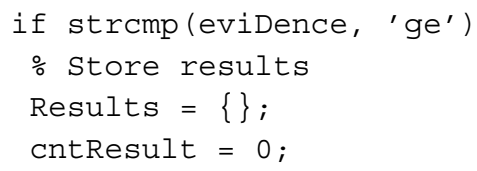

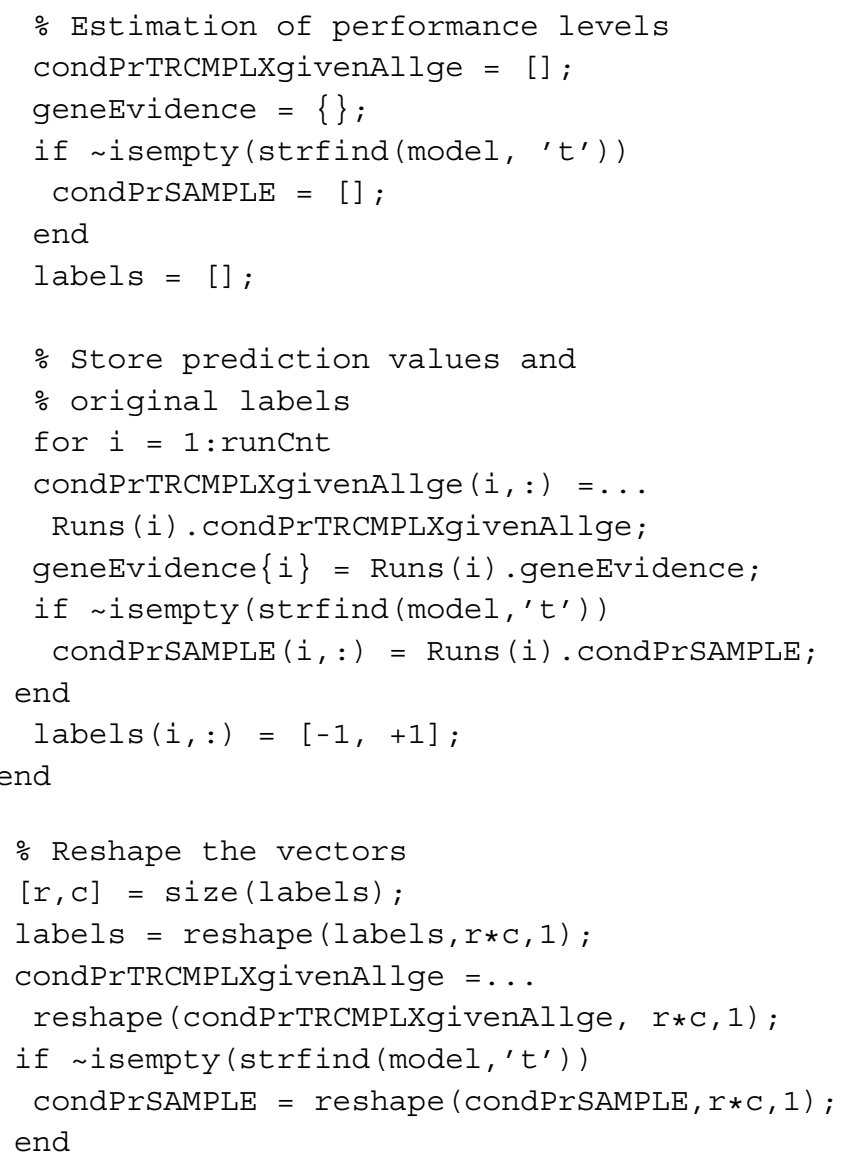




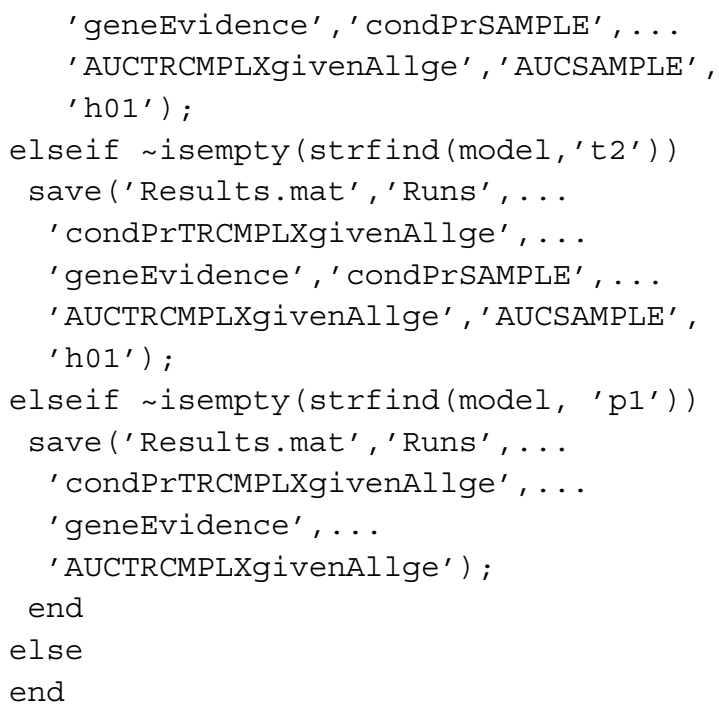

The ROC graphs and their respective AUC values found in the figures of [1] are plotted by making variation in the assumed probability values of PrTRCMPLX in the function generateGenecpd. The details of the generateGenecpd are discussed in the next section.

The variation in the assumed probability values of the TRCMPLX that affect the behavior of the gene nodes is termed as ETGN in [1]. Since the entire code runs only once, it has to be run for different instances of input arguments, separately. Once the results have been saved in Results.mat file, one can rename the file based on the model and the evidence arguments used in function twoHoldOutExp. Thus, if the code is run for model " $t 1$ " and ETGN of $90 \%$, then the user needs to rename the Results.mat that stores the results with an appropriate file name like Results-T1-GE-pforTRCMPLX-90per.mat. Once the results for all permutations of instances for a vector of input arguments in twoHoldOutExp have been obtained, the script geneTRCMPLXstats using the generated mat result files can be executed to generate the tables which shows how TRCMPLX behaves as the evidences of genes vary in both normal and tumorous cases. Tables 5 and 6 in [1] are generated using this script. How interpretations of the results are made can be studied in more depth in the results section of [1]. However, succinctly, the script geneTRCMPLXstats generates mean/average estimates of the conditional probability that the transcription complex will be switched on or off in normal or tumor test samples, given the different gene evidences. By majority, if a gene expression is found to be repressed (active) in normal or tumor case, then the predicted belief represented by the probability of the transcription complex conditional on repression (activation) is chosen as the inferred biological phenomena. Figures 6 and 7 of [1] depict the summarized pictorial representation of the predicted inferences shown in Tables 5 and 6 of [1].
Note that to generate the $\mathrm{ROC}$ graphs and their respective AUC values for different models with varying effect of TRCMPLX on different genes (ETGN in [1]), the results in variables $X$ and $Y$ (of twoHoldOutExp) are stored in different variables and clumped together in a .mat file titled aucANDpredictions_sample_TRCMPLX.mat. This has to be done manually for each model and every setting of ETGN. For example, using model t1 and ETGN of $60 \%$, the false positive rate in X is stored as XT1_60 and the true positive rate in $\mathrm{Y}$ is stored as $\mathrm{YT}^{\mathrm{T}}{ }_{-} 60$, in the abovementioned .mat file. Finally, the script in the $m$ file titled plotAUC is used to manipulate the aforementioned transformed variables and generate the ROC curves in Figure 5 of the results section of [1]. The Google drive https://drive.google.com/folderview?id=0B7Kkv8wlhPUT05wTTNodWNydjA\&usp=sharing contains the results under the compressed directory with name Results-2013. Inference interpretations of the results can be studied in more depth from [1].

Finally, a full section is dedicated to the computation of the probabilities for nodes with parents which has been implemented in function generateGenecpd. The computation of gene nodes happens within the holdout experiment and before the new computation of CPTs conditional on the provided evidence. Since the details of computation of CPTs for gene nodes is dense, it has been treated separately after the explanation of the code of holdout experiment.

\subsection{Generating probabilities for gene nodes with parents}

Here, the code for the function generateGenecpd is explained. As a recapitulation, the function generateGenecpd in the script generateGenecpd.m takes the following input arguments: (1) vecTraining-gene expression from the training data, (2) label Training-labels of the training data, (3) nodeName-name of the gene involved, (4) parent-name of parents of the child node or the gene under consideration, (5) parent_cpd-parent cpd values, (6) model-kind of the model and finally returns the output as a structure gene_cpd containing cpd for the particular gene under consideration given its parents as well as a threshold value in the form of median. In the code below, the values of the following variables are used as input arguments for the function generateGenecpd, in order: (1) dataForTraining $(1,:)$-training data for the $l$ th unique gene, (2) labelForTraininglabels for the training data, (3) uniqueGenes $\{1\}$, (4) parent, (5) parent_cpd, (6) model. The output of the function is stored in the structure variable $x$. The threshold at which the probabilities were computed for the $l$ th gene is stored in gene_cpd ( 1 ). vecmedian using $\mathrm{x}$.vecmedian and the probabilities themselves are stored in gene_cpd ( 1 ). T using X.T. 
The code begins with the storing of the dimension of a gene expression vector in vecTraining in variables $r$ and $\mathrm{C}$ and recording the length of the vector containing the labels of the training data (in labelTraining) in variable lencond. Finally, the much reported threshold is estimated here using the median of the training data and stored in vecmedian.

$\%$ Rows is the gene expression and...

\% columns are conditions (normal or

$\%$ cancerous) $[r, c]=$ size (vecTraining);

$\%$ lencond = length (labelTraining);

\% Take median as the threshold vecmedian =

o median (vecTraining);

In [1], the effect of TRCMPLX on the gene expression has been analyzed as it is not known to what degree the TRCMPLX plays a role in the Wnt signaling pathway. To investigate this, Sinha [1] incorporated a parameter $\mathrm{p}$ that encodes the effect of TRCMPLX on the expression of the gene which is influenced by it. Thus, while iterating through the list of parents if one encounters TRCMPLX as a parent, then $\mathrm{p}$ is initialized to a certain value. In [1], the effect of TRCMPLX being active $(1-p)$ is incremented in steps of 0.1 from $\{0.5$ to 0.9$\}$ and respective $\mathrm{ROC}$ graphs are plotted using the same.

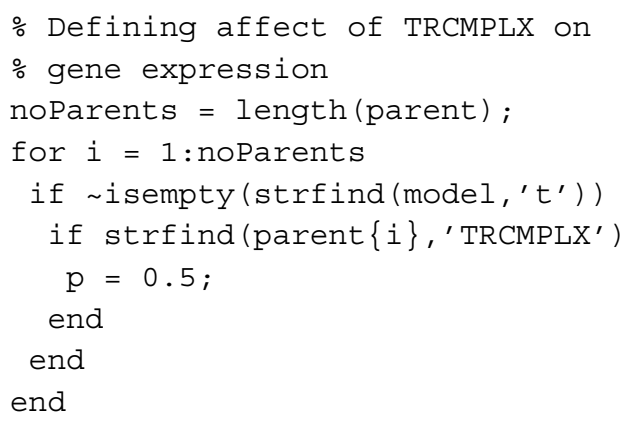

It is important to note that the computation of gene probabilities differ from model to model and a detailed description of each computation is given for each gene for all three models, before explaining the computation for another gene. Also, from [1], theoretically, for a gene $g_{i} \forall i$ genes, let there be $n_{\mathrm{tr}}$ different instances of expression values from the sample training data. Let each of the $n_{\text {tr }}$ gene expression values be discretized to 0 and 1 based on their evaluation with respect to the median threshold. The 1's represent the total number of expression where the gene is active and 0's represent the total number of expression where the gene is inactive. In case of normal and tumorous samples, the proportions of 1's and 0's may be different. The median of the expression values is employed as a threshold to decide the frequency of $g_{i}$ being active or inactive given the state of the parent node(s). This median is also used along with the labels of the training data to decide the status of different parent factors affecting the gene under consideration.

If one observes the network in Figs. 2 and 4, one finds that there are nodes that have one, two, or three parent nodes. Computation of conditional probability tables for these child nodes which represent gene expression for both tumor and normal samples in the different models (i.e., " 11 " for $\mathcal{M}_{\mathrm{PBK}+\mathrm{EI}}$, " $\mathrm{t} 2$ " for $\mathcal{M}_{\mathrm{PBK}}$, and "p1" for $\left.\mathcal{M}_{\mathrm{NB}+\mathrm{MPBK}}\right)$ require intuitive analysis of the expression data. Estimation of the cpts for three gene nodes, i.e., $D K K 1, D K K 2$, and $D A C T 3$, each having different parents depending on the type of the model has been explained below. Nodes that have similar corresponding behavior are enlisted but the estimation is not derived.

\subsubsection{DKK1 in $\mathcal{M}_{P B K+E I}(t 1)$}

Since there are three parents for $D K K 1$, namely $M e D K K 1$, Sample, and TRCMPLX, the cpt values for the table is segregated based on the status of methylation and quality of samples. A $2 \times 2$ cross table for methylation and sample generates frequency estimates that can help derive probability values. The entries of the cross table depict the following cases: (a) methylated in normal (represented by vector mINn), (b) un-methylated in normal (represented by vector umINn), (c) methylated in tumorous (represented by vector mINt), and (d) un-methylated in tumorous (represented by vector umINt) cases. For every $j$ th entry in the vecTraining, if the label (labelTraining $(j))$ is normal $(\leq 0)$ and the $D K K 1$ gene expression (vecTraining $(j))$ is less than the estimated median ( $\leq$ vecmedian), then the value in vecTraining $(j)$ is appended to mINn. Here, expression level lower than median indicates probable repression due to methylation in normal case. If the label (labelTraining $(j))$ is normal $(\leq 0)$ and the $D K K 1$ gene expression (vecTraining $(j))$ is greater than the estimated median ( $\geq$ vecmedian), then the value in vecTraining $(j)$ is appended to umINn. Here, expression level greater than median indicates probable activation due to un-methylation in normal case. If the label (labelTraining $(j)$ ) is tumorous $(\geq 0)$ and the $D K K 1$ gene expression (vecTraining $(j))$ is less than the estimated median ( $\leq$ vecmedian), then the value in vecTraining $(j)$ is appended to mINt. Here, expression level lower than median indicates probable repression due to methylation in tumorous case. And finally, if the label (labelTraining $(j)$ ) is tumorous $(\geq 0)$ and the $D K K 1$ gene expression (vecTraining $(j))$ is greater than the estimated median ( $\geq$ vecmedian), then the value in vecTraining $(j)$ is appended to umINt. Here, expression level greater than median indicates probable activation due to un-methylation in tumorous case. 


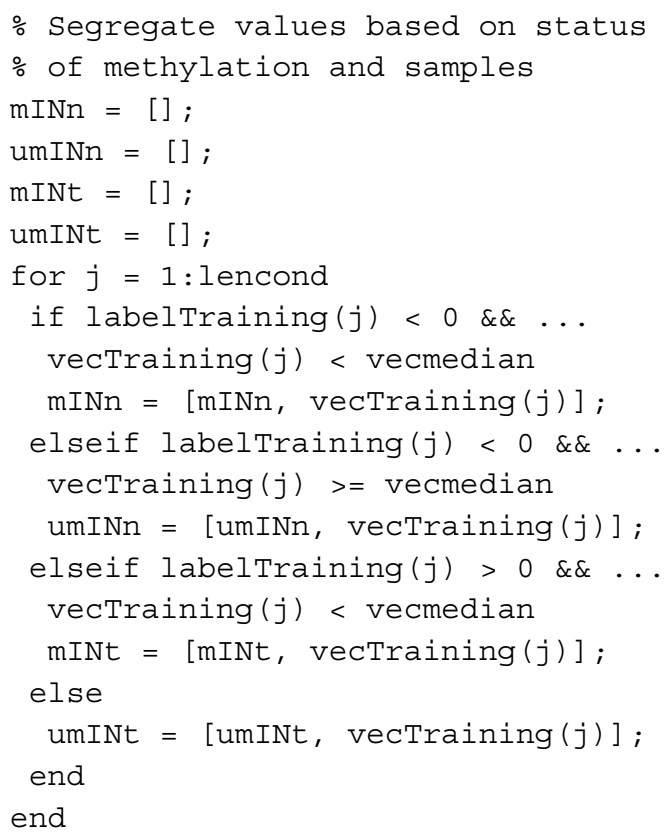

Also, since the actual probability values for the activation of the TRCMPLX is not known, the conditional probabilities are multiplied with a probability value of $p$ when the TRCMPLX is off and with a probability value $1-p$ when the TRCMPLX is on. Before estimating the values for cpt of $D K K 1$, it is important to see how (1) the probability table would look like and (2) the probability table is stored in BNT [4]. Table 10 represents the conditions of sample as well as the methylation along with transcription complex and the probable beliefs of events (DKK1 being on/off). With three parents and binary state, the total number of conditions is $2^{3}$. To estimate the values of the probable beliefs of an event, the following computation is done. (Case - TRCMPLX is Off) The $\operatorname{Pr}(D K K 1$ - On|Sample - Normal, Me - UM) being low is the fraction of number of 1's in the normal sample

Table 10 Conditional probability table for DKK1 in $\mathcal{M}_{\mathrm{PBK}+\mathrm{EI}}$ (model - t1)

\begin{tabular}{|c|c|c|c|c|}
\hline \multicolumn{5}{|c|}{ CPT for DKK 1 in $\mathcal{M}_{\text {PBK+EI }}($ model - $\mathrm{t} 1)$} \\
\hline Sample & Methylation & TRCMPLX & $\operatorname{Pr}(D K K 1=\mathrm{Off})$ & $\operatorname{Pr}(D K K 1=O n)$ \\
\hline Normal & No & Off & h (1) & I (9) \\
\hline Tumor & No & Off & $h / l(2)$ & $\mathrm{I} / \mathrm{h}(10)$ \\
\hline Normal & Yes & Off & h (3) & I (11) \\
\hline Tumor & Yes & Off & h (4) & I (12) \\
\hline Normal & No & On & h (5) & I (13) \\
\hline Tumor & No & On & $h / l(6)$ & $\mathrm{l} / \mathrm{h}(14)$ \\
\hline Normal & Yes & On & h (7) & I (15) \\
\hline Tumor & Yes & On & h (8) & I (16) \\
\hline
\end{tabular}

h - probability of event being high; I - probability of event being low. Serial numbers in brackets represent the ordering of numbers in vectorial format $(a \times p)$ and the sum of total number of normal samples and number of 1's in the tumorous samples, i.e., the nonmethylated gene expression values in tumorous samples (A). Similarly, $\operatorname{Pr}(D K K 1$ - On|Sample - Tumor, Me - UM) being low is the fraction of number of 1's in the tumorous sample $(b \times p)$ and the sum of total number of tumorous samples and number of 1's in the normal samples, i.e., the non-methylated gene expression values in normal samples (B). Again, $\operatorname{Pr}(D K K 1$ - Off $\mid$ Sample - Normal, $M e-\mathrm{M})$ being high is the fraction of number of 0's in the normal sample $(\mathrm{c} \times p)$ and the sum of total number of normal samples and number of 0's in the tumorous samples, i.e., the methylated gene expression values in tumorous samples (C). Finally, $\operatorname{Pr}(D K K 1$ - Off $\mid$ Sample - Tumor, $M e$ - M) being high is the fraction of number of 0's in the tumorous sample $(\mathrm{d} \times p)$ and the sum of total number of tumorous samples and number of 0 's in the normal samples, i.e the methylated gene expression values in normal samples (D).

(Case - TRCMPLX is On) Next, the $\operatorname{Pr}(D K K 1$ On|Sample - Normal, $M e$ - UM) being low is the fraction of number of 1's in the normal sample $(a \times(1-p))$ and the sum of total number of normal samples and number of 1's in the tumorous samples, i.e., the non-methylated gene expression values in tumorous samples (A). Similarly, $\operatorname{Pr}(D K K 1$ - On|Sample - Tumor, Me - UM) being low is the fraction of number of 1's in the tumorous sample $(\mathrm{b} \times(1-p))$ and the sum of total number of tumorous samples and number of 1's in the normal samples, i.e., the non-methylated gene expression values in normal samples (B). Again, $\operatorname{Pr}(D K K 1$ - Off $\mid$ Sample - Normal, $M e$ - M) being high is the fraction of number of 0's in the normal sample $(c \times(1-p))$ and the sum of total number of normal samples and number of 0 's in the tumorous samples, i.e., the methylated gene expression values in tumorous samples (C). Finally, $\operatorname{Pr}(D K K 1$ - Off $\mid$ Sample - Tumor, Me - M) being high is the fraction of number of 0's in the tumorous sample $(\mathrm{d} \times(1-p))$ and the sum of total number of tumorous samples and number of 0's in the normal samples, i.e., the methylated gene expression values in normal samples (D). Complementary conditional probability values for $D K K 1$ being inactive can easily be computed from the above estimated values.

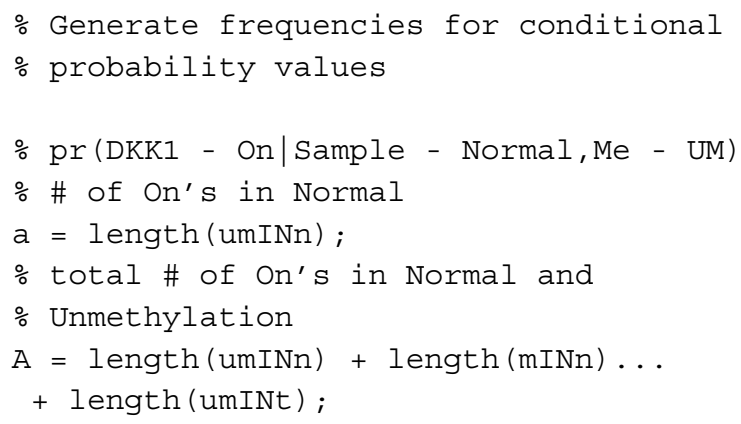


\% pr (DKK1 - On|Sample - Tumor, Me - UM)

\% \# of On's in Tumor

$\mathrm{b}=$ length (umINt);

\% total \# of On's in Normal and

\% Unmethylation

$\mathrm{B}=$ length (umINt) + length (umINn) ...

+ length (mINt);

\% pr(DKK1 - Off|Sample - Normal, Me - M)

$\%$ \# of Off's in Normal

$\mathrm{C}=$ length (mINn);

\% total \# of Off's in Normal and...

\% Methylation

$\mathrm{C}=$ length (mINn) + length (umINn) $\ldots$

+ length (mINt);

\% pr(DKK1 - Off|Sample - Tumor, Me - M)

\% \# of Off's in Normal

$\mathrm{d}=$ length (mINt);

\% total \# of Off's in Normal and

\% Methylation

$\mathrm{D}=$ length (mINt) + length (umINt) ...

+ length (mINn);

These values are stored in variable $\mathrm{T}$ and the estimation is shown in the following section of the code. After the values in $\mathrm{T}$ have been established, a constant 1 is added as pseudo-count to convert the distribution to a probability distribution via Dirichlet process. This is done to remove any deterministic $0 / 1$ values appearing in the probability tables. If $0 / 1$ appears in the probability tables then one has deterministic evidence regarding an event and the building of the Bayesian engine collapses. These counts also represent the unobserved that might not have been recorded due to small sample size. The Dirichlet process is a generalization of the Dirichlet distribution which is parameterized by a vector of positive reals. The pseudocounts here form the positive values. What this basically means is that the probability density function returns the belief that the probabilities of some rival events given that each event has been observed non-negative number of times. These distributions are often used as prior distributions in Bayesian statistics.

Finally, the frequencies/probabilities in $\mathrm{T}$ are normalized in order to obtain the final conditional probability values for $D K K 1$. Estimation of cpts for genes SFRP1, WIF 1 and DKK 4 which have methylation, TRCMPLX and Sample as parents require same computations as above. Figure 5 shows the pictorial representation of one of the cpt in $\mathcal{M}_{\mathrm{PBK}+\mathrm{EI}}$.

\% Multiply probability of TRCMPLX in

\% on/off state to add the $3 r d$

\% dimension in deciding the conditional

\% probability tables.

\% Conditional probability table for

\% DKK1 given its parents

$\mathrm{T}=[\mathrm{A}-\mathrm{a} * \mathrm{p}, \mathrm{a} * \mathrm{p} ; \ldots$

$\mathrm{B}-\mathrm{b} * \mathrm{p}, \mathrm{b} * \mathrm{p} ; \ldots$

$\mathrm{C} * \mathrm{p}, \mathrm{C}-\mathrm{C} * \mathrm{p} ; \ldots$

$d * p, \quad D-d * p ; \ldots$

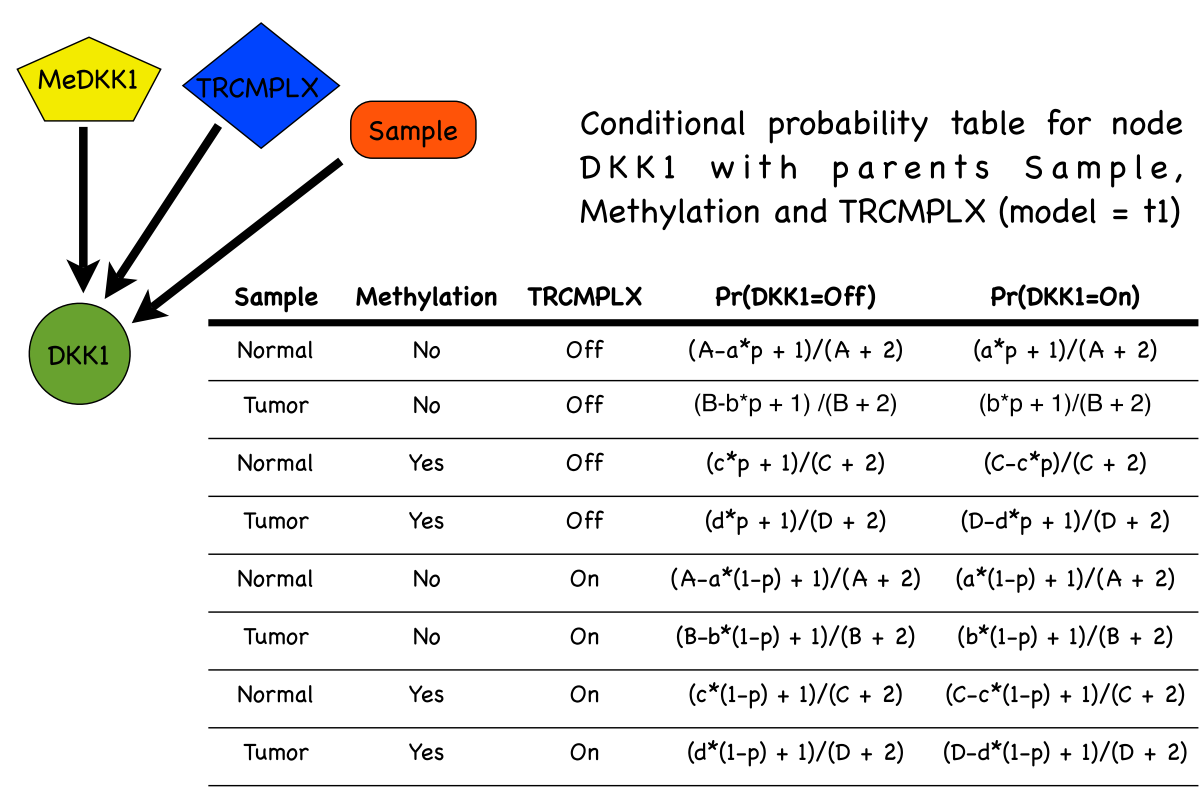

Fig. 5 Conditional probability table for node DKK1 in $\mathcal{M}_{P B K+E l}$ 


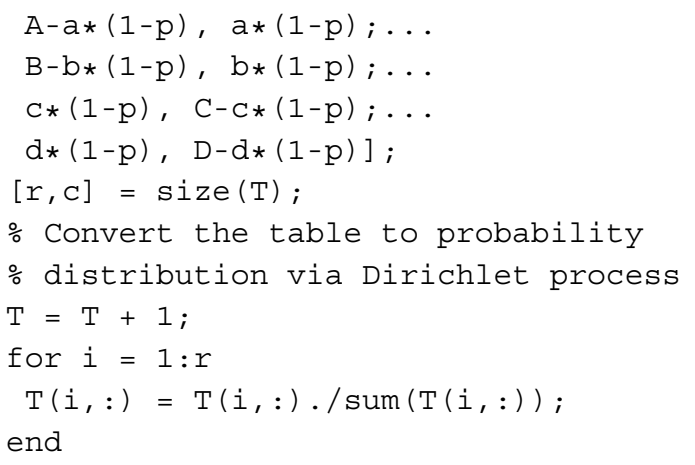

\subsubsection{DKK1 in $\mathcal{M}_{P B K}(t 2)$}

There are two parents for $D K K 1$, namely TRCMPLX and Sample. The conditional probability value for a gene being active or inactive is estimated based on the state of the Sample. Again, since the actual probability values for the activation of the TRCMPLX is not known the conditional probabilities are multiplied with a probability value of $p$ when the TRCMPLX is off and with probability value $1-p$ when the TRCMPLX is on.

The analysis of quality of sample generates frequency estimates that can help derive probability values. These frequencies depict the following cases: (a) gene repressed in normal (represented by vector offINn), (b) gene expressed in normal (represented by vector onINn), (c) gene repressed in tumorous (represented by vector offINt), and (d) gene expressed in tumorous (represented by vector onINt) cases. For every $j$ th entry in the vecTraining, if the label (labelTraining $(j)$ ) is normal $(\leq 0)$ and the $D K K 1$ gene expression (vecTraining $(j)$ ) is less than the estimated median ( $\leq$ vecmedian), then the value in vecTraining $(j)$ is appended to offINn. Here, expression level lower than median indicates probable gene repression in the normal case. If the label (labelTraining $(j)$ ) is normal $(\leq 0)$ and the $D K K 1$ gene expression (vecTraining $(j)$ ) is greater than the estimated median ( $\geq$ vecmedian), then the value in vecTraining $(j)$ is appended to onINn. Here, expression level greater than median indicates probable gene activation in normal case. If the label (labelTraining $(j)$ ) is tumorous $(\geq 0)$ and the $D K K 1$ gene expression (vecTraining $(j)$ ) is less than the estimated median ( $\leq$ vecmedian), then the value in vecTraining $(j)$ is appended to offInt. Here, expression level lower than median indicates probable gene repression in tumor case. And finally, If the label (labelTraining $(j)$ ) is tumorous $(\geq 0)$ and the $D K K 1$ gene expression (vecTraining $(j)$ ) is greater than the estimated median ( $\geq$ vecmedian), then the value in vecTraining $(j)$ is appended to onINt. Here, expression level greater than median indicates probable gene activation in tumorous case.

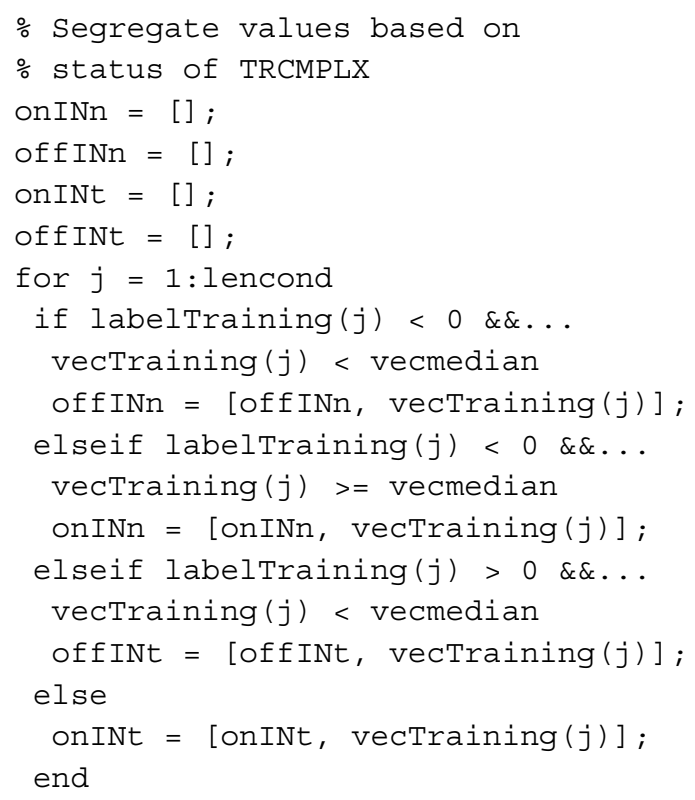
end

Again, before estimating the values for cpt of $D K K 1$, it is important to see how (1) the probability table would look like and (2) the probability table is stored in BNT [4]. Table 11 represents the conditions of Sample as well as TRCMPLX and the probable beliefs of events (DKK1 being on/off). With two parents and binary state, the total number of conditions is $2^{2}$. To estimate the values of the probable beliefs of an event, the following computation is done. The probability of gene expression being active given Sample is normal and TRCMPLX is off, i.e., $\operatorname{Pr}(D K K 1=$ Active $\mid$ Sample $=$ Normal, TRCMPLX = Off $)$, is the fraction of number of 1's in the normal sample $(a \times p)$ and the sum of total number of normal samples (A). Similarly, the probability of gene expression being active given Sample is tumorous and TRCMPLX is off, i.e., $\operatorname{Pr}(D K K 1=$ active $\mid$ Sample = tumorous, TRCMPLX $=$ Off), is the fraction of number of 1's in the tumorous sample $(\mathrm{b} \times p)$ and the sum of total number of tumorous samples (B). Again, the probability of gene expression being inactive given Sample is normal and TRCMPLX is

Table 11 Conditional probability table for DKK 1 in $\mathcal{M}_{\mathrm{PBK}}$ (model - t2)

\begin{tabular}{llll}
\hline CPT for DKK1 in $\mathcal{M}_{\text {PBK }}($ model $-\mathrm{t} 2)$ & & \\
\hline Sample & TRCMPLX & $\operatorname{Pr}(D K K 1=$ Off $)$ & $\operatorname{Pr}(D K K 1=$ On $)$ \\
\hline Normal & Off & $\mathrm{h}(1)$ & $\mathrm{I}(5)$ \\
Tumorous & Off & $\mathrm{I}(2)$ & $\mathrm{h}(6)$ \\
Normal & On & $\mathrm{h}(3)$ & $\mathrm{I}(7)$ \\
Tumorous & On & $\mathrm{I}(4)$ & $\mathrm{h}(8)$ \\
\hline
\end{tabular}

$\mathrm{h}$ - probability of event being high; I - probability of event being low. Serial numbers in brackets represent the ordering of numbers in vectorial format 
on, i.e., $\operatorname{Pr}(D K K 1=$ inactive $\mid$ Sample $=$ normal, $T R C M P L X$ $=\mathrm{On}$ ), is the fraction of number of 0's in the normal sample $(\mathrm{A}-\mathrm{a} \times(1-p))$ and the sum of total number of normal samples (A). Lastly, the probability of gene expression being inactive given Sample is tumorous and TRCM$P L X$ is on, i.e., $\operatorname{Pr}(D K K 1=$ inactive $\mid$ Sample = tumorous, TRCMPLX = On), is the fraction of number of O's in the tumorous sample $(\mathrm{B}-\mathrm{b} \times(1-p))$ and the sum of total number of tumorous samples (b). Complementary conditional probability values for $D K K 1$ being inactive can easily be computed from the above estimated values.

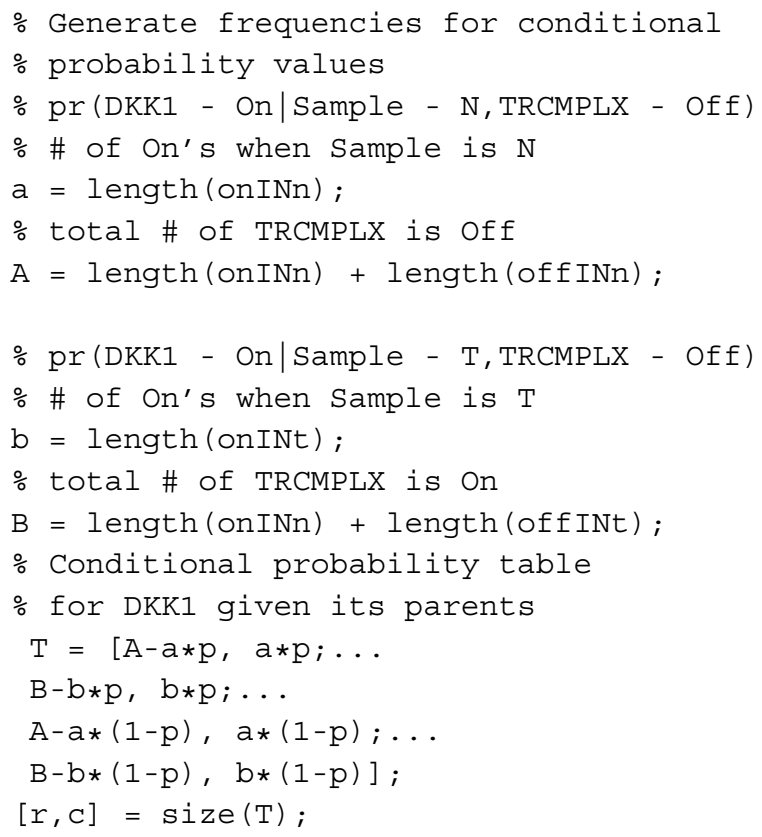

After the values in $\mathrm{T}$ have been established, a constant 1 is added as pseudo-count to convert the distribution to a probability distribution via Dirichlet process. Finally, the frequencies in $\mathrm{T}$ are normalized in order to obtain the final conditional probability values for $D K K 1$. Estimation of cpts for genes SFRP1, CCND1, CD44, WIF1, MYC, and DKK4 which has TRCMPLX and Sample as parents requires the same computations as above. Figure 6 shows the pictorial representation of one of the cpt in $\mathcal{M}_{\text {РВK. }}$

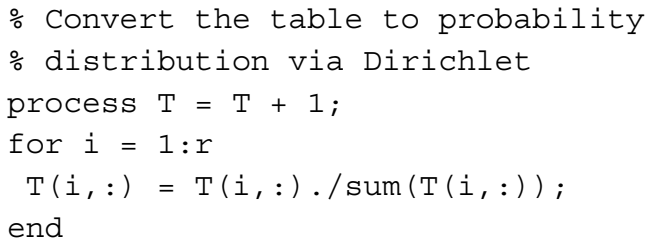

\subsubsection{DKK1 in $\mathcal{M}_{N B+M P B K}(p 1)$}

Following the naive Bayes model presented by [3] and making slight modifications to it, Sinha [1] generated $\mathcal{M}_{\mathrm{NB}+\mathrm{MPBK}}$. In this, all genes have a single parent, namely TRCMPLX, and it is assumed that the predicted state of TRCMPLX is exactly the same as the quality of the test sample. Thus, the initial probability values for TRCM$P L X$ are assumed to be fixed and no variation is made on it. The conditional probability value for a gene being active or inactive is estimated based on the state of the TRCMPLX.

The segregation of the probability values depends on the following conditions: (a) gene is active and TRCM$P L X$ is on (represented by vector onINTrOn), (b) gene is inactive and TRCMPLX is off (represented by vector offINTron), (c) gene is active and TRCMPLX is off (represented by vector onINTrOff), and (d) gene is inactive (represented by vector offINTrOff). For every $j$ th entry in the vecTraining, if the label (labeltraining $(j)$ ) is $\leq 0$ (TRCMPLX is off) and the $D K K 1$ gene expression (vecTraining $(j)$ ) is less than the estimated median ( $\leq$ vecmedian), then the value in vecTraining $(j)$ is appended to offINTroff. If the label (labelTraining $(j))$ is $\leq 0$ (TRCMPLX is off) and the $D K K 1$ gene expression (vecTraining $(j)$ ) is greater than the estimated median ( $\geq$ vecmedian), then the value in vecTraining $(j)$ is appended to onINTroff. If the label (labelTraining $(j)$ ) is $\geq 0$ (TRCMPLX is on) and the DKK1 gene expression (vecTraining $(j)$ ) is less than the estimated median

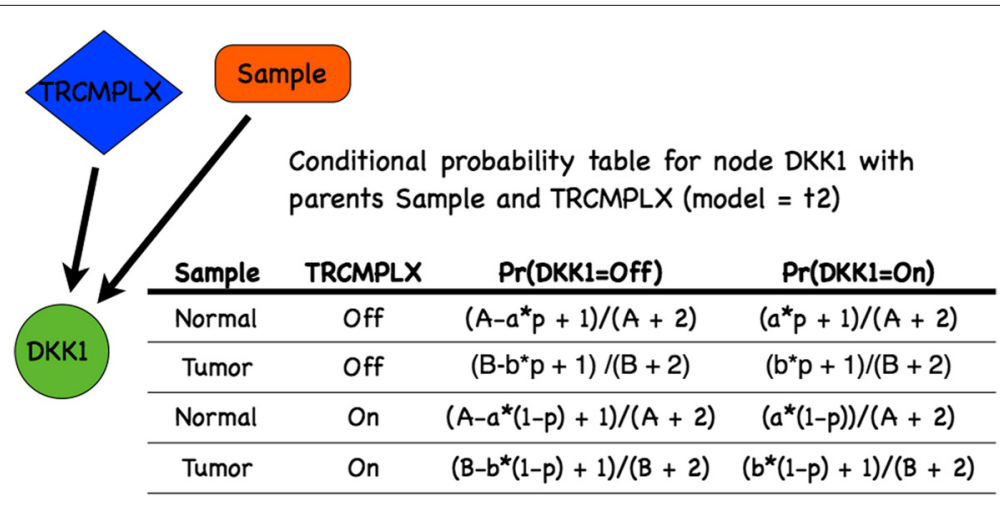

Fig. 6 Conditional probability table for node DKK1 in $\mathcal{M}_{P B K}$ 
( $\leq$ vecmedian), then the value in vecTraining $(j)$ is appended to offINTrOn. And finally, if the label (labelTraining $(j)$ ) is $\geq 0$ (TRCMPLX is on) and the $D K K 1$ gene expression (vecTraining $(j))$ is greater than the estimated median ( $\geq$ vecmedian), then the value in vecTraining $(j)$ is appended to onINTrOn.

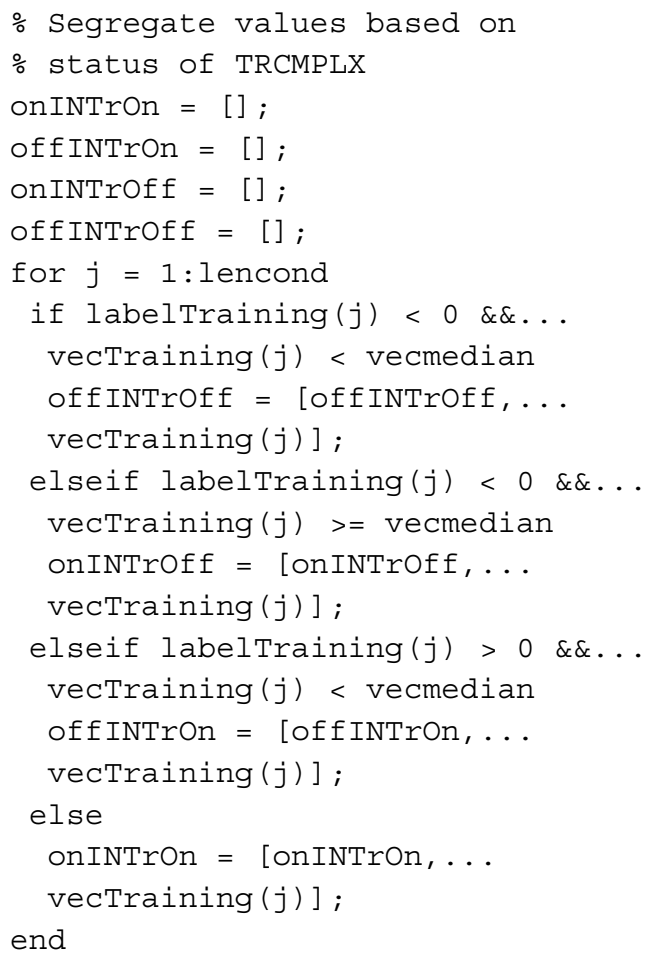

Lets again see how (1) the probability table would look like and (2) the probability table is stored in BNT [4] before estimating the values for cpt of $D K K 1$. Table 12 represents the conditions of TRCMPLX and the probable beliefs of events (DKK1 being on/off). With a single parent and binary state, the total number of conditions is $2^{1}$. To estimate the values of the probable beliefs of an event, the following computation is done. The probability of gene expression being active given TRCMPLX is off, i.e., $\operatorname{Pr}(D K K 1=$ Active $\mid T R C M P L X=$ Off $)$, is the fraction of number of 1's in the normal sample (a) and the sum of total number of normal samples (A). Similarly, the probability of gene expression being inactive given TRCMPLX

Table 12 Conditional probability table for DKK 1 in $\mathcal{M}_{\mathrm{NB}+\mathrm{MPBK}}$ (model-p1)

\begin{tabular}{lll}
\hline \multicolumn{2}{l}{ CPT for DKK1 in $\mathcal{M}_{\mathrm{NB}+\mathrm{PBK}}($ model - $\mathrm{p} 1)$} & \\
\hline TRCMPLX & $\operatorname{Pr}(D K K 1=\mathrm{Off})$ & $\operatorname{Pr}(\mathrm{DKK} 1=\mathrm{On})$ \\
\hline Off & $\mathrm{h}(1)$ & I (3) \\
On & $\mathrm{h}(2)$ & I (4) \\
\hline
\end{tabular}

$\mathrm{h}$ - probability of event being high; I - probability of event being low. Serial numbers in brackets represent the ordering of numbers in vectorial format is off, i.e., $\operatorname{Pr}(D K K 1=$ active $\mid T R C M P L X=$ On $)$, is the fraction of number of 1's in the tumorous sample (b) and the sum of total number of tumorous samples (B). Complementary conditional probability values for $D K K 1$ being inactive can easily be computed from the above estimated values. Figure 6 shows the pictorial representation of one of the cpt in $\mathcal{M}_{\text {PBK. }}$.

\% Generate frequencies for

\% conditional probability values

\% $\operatorname{pr}(D K K 1$ - On | TRCMPLX - off)

$\%$ \# of On's when TRCMPLX is off

$\mathrm{a}=$ length (onINTroff);

\% total \# of TRCMPLX is off

$A=$ length (onINTroff) + length (offINTroff);

\% pr (DKK1 - On | TRCMPLX - On)

\% \# of On's when TRCMPLX is On

$\mathrm{b}=$ length (onINTrOn);

\% total \# of TRCMPLX is on

$\mathrm{B}=$ length (onINTron) + length (offINTron);

\% Conditional probability table

\% for DKKI given its parents

$\mathrm{T}=[\mathrm{A}-\mathrm{a}, \mathrm{a} ; \ldots$

$\mathrm{B}-\mathrm{b}, \mathrm{b}]$;

$[r, \mathrm{c}]=\operatorname{size}(\mathrm{T})$;

After the values in $\mathrm{T}$ have been established, a constant 1 is added as pseudo count to convert the distribution to a probability distribution via the Dirichlet process. Finally, the frequencies in $\mathrm{T}$ are normalized in order to obtain the final conditional probability values for $D K K 1$. Figure 7 shows the pictorial representation of one of the cpt in $\mathcal{M}_{\mathrm{NB}+\mathrm{MPBK}}$.

\% Convert the table to probability

\% distribution via Dirichlet process

$\% \mathrm{~T}=\mathrm{T}+1 ;$ for $i=1: r$

$\mathrm{T}(i,:)=\mathrm{T}(i,:) \cdot / \operatorname{sum}(\mathrm{T}(i,:))$;

end

\subsubsection{DKK2 in $\mathcal{M}_{P B K+E I}(t 1)$}

The Sample is the single parent of $D K K 2$. The conditional probability value for a gene being active or inactive is estimated based on the state of the Sample. The analysis of quality of sample generates frequency estimates that can help derive probability values. These frequencies depict the following cases: (a) gene repressed in normal (represented by vector offINn), (b) gene expressed in normal (represented by vector onINn), (c) gene repressed in tumorous (represented by vector offINt), and (d) gene expressed in tumorous (represented by vector onINt) cases. For every $j$ th entry in the vecTraining, if the label (labelTraining $(j))$ is normal $(\leq 0)$ and 


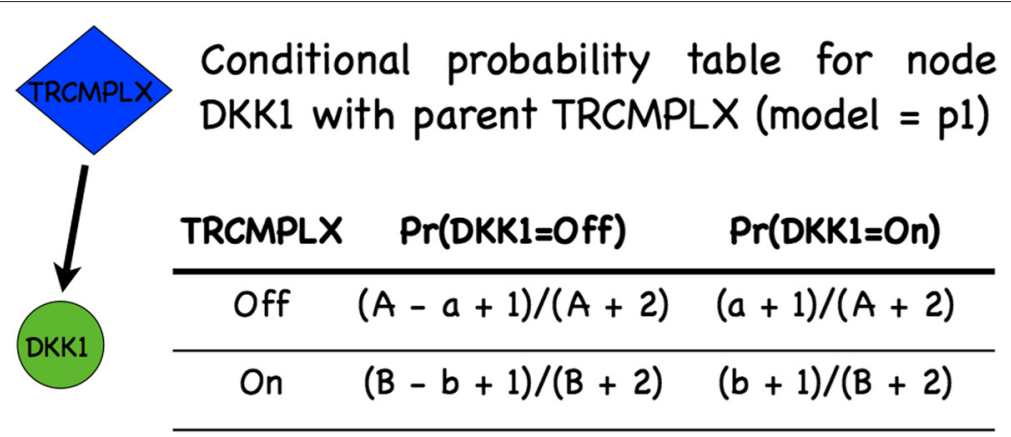

Fig. 7 Conditional probability table for node DKK 1 in $\mathcal{M}_{\mathrm{NB}+\mathrm{MPBK}}$

the DKK2 gene expression (vecTraining $(j)$ ) is less than the estimated median ( $\leq$ vecmedian), then the value in vecTraining $(j)$ is appended to offINn. Here, expression level lower than median indicates probable gene repression in the normal case. If the label (labelTraining $(j)$ ) is normal $(\leq 0)$ and the $D K K 2$ gene expression (vecTraining $(j))$ is greater than the estimated median ( $\geq$ vecmedian), then the value in vecTraining $(j)$ is appended to onINn. Here, expression level greater than median indicates probable gene activation in the normal case. If the label (labelTraining $(j)$ ) is tumorous $(\geq 0)$ and the $D K K 2$ gene expression (vecTraining $(j))$ is less than the estimated median ( $\leq$ vecmedian), then the value in vecTraining $(j)$ is appended to offINt. Here, expression level lower than median indicates probable gene repression in the tumor case. And finally, If the label (labelTraining $(j)$ ) is tumorous $(\geq 0)$ and the $D K K 2$ gene expression (vecTraining $(j))$ is greater than the estimated median ( $\geq$ vecmedian), then the value in vecTraining $(j)$ is appended to onINt. Here, expression level greater than median indicates probable gene activation in the tumorous case.

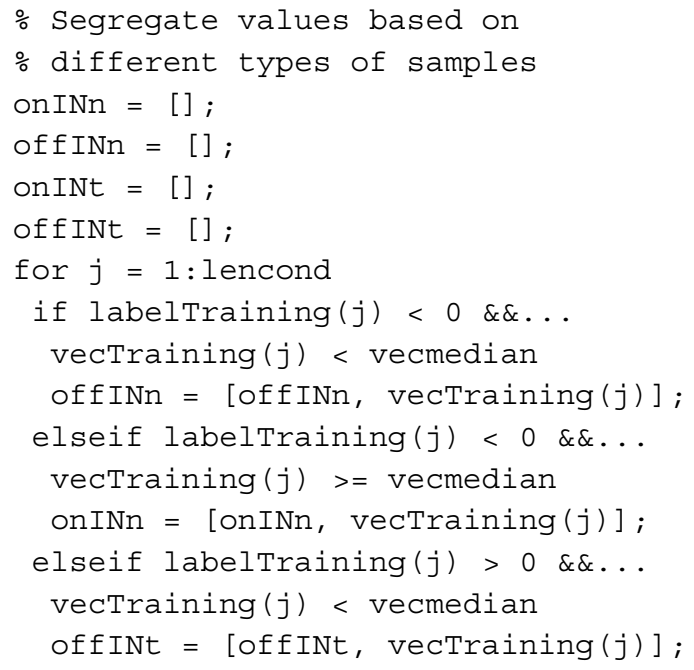

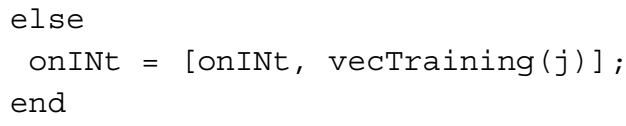

Lets again see how (1) the probability table would look like and (2) the probability table is stored in BNT [4] before estimating the values for cpt of $D K K 2$. Table 13 represents the conditions of Sample and the probable beliefs of events ( $D K K 2$ being on/off). With a single parent and binary state, the total number of conditions is $2^{1}$. To estimate the values of the probable beliefs of an event, the following computation is done. The probability of gene expression being active given Sample is normal, i.e., $\operatorname{Pr}(D K K 2=$ Active $\mid$ Sample $=$ Normal $)$, is the fraction of number of 1's in the normal sample (a) and the sum of total number of normal samples (A). Similarly, the probability of gene expression being active given Sample is tumorous, i.e., $\operatorname{Pr}(D K K 2=$ active $\mid$ Sample = Tumorous $)$, is the fraction of number of 1's in the tumorous sample (b) and the sum of total number of tumorous samples (B). Complementary conditional probability values for $D K K 2$ being inactive can easily be computed from the above estimated values.

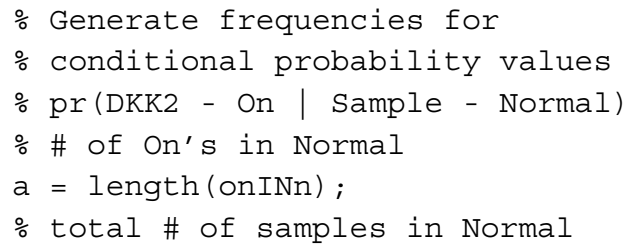

Table 13 Conditional probability table for DKK2 in $\mathcal{M}_{\text {NB+MPBK }}$ (model - t1)

\begin{tabular}{lll}
\hline CPT for DKK2 in $\mathcal{M}_{\text {NB+PBK }}($ model $-\mathrm{t} 1)$ & \\
\hline Sample & $\operatorname{Pr}(D K K 2=$ Off $)$ & $\operatorname{Pr}(D K K 2=\mathrm{On})$ \\
\hline Normal & $\mathrm{l} / \mathrm{h}(1)$ & $\mathrm{h} / \mathrm{l}(3)$ \\
Tumor & $\mathrm{h} / \mathrm{l}(2)$ & $\mathrm{l} / \mathrm{h}(4)$ \\
\hline
\end{tabular}

$\mathrm{h}$ - probability of event being high; I - probability of event being low. Serial numbers in brackets represent the ordering of numbers in vectorial format 


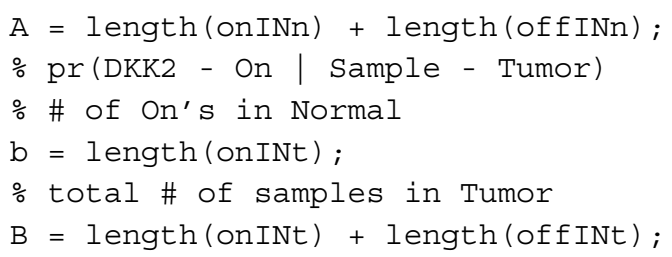

After the values in $\mathrm{T}$ have been established, a constant 1 is added as pseudo-count to convert the distribution to a probability distribution via Dirichlet process. Finally, the frequencies in $\mathrm{T}$ are normalized in order to obtain the final conditional probability values for $D K K 2$. Estimation of cpts for genes $D K K 3-1, D K K 3-2$, SFRP3, and $L E F 1$ which have Sample as parent requires the same computations as above.

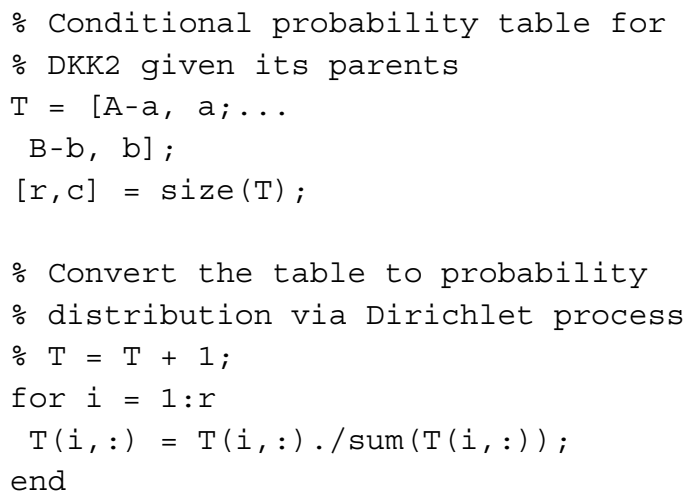

\subsubsection{DKK2 in $\mathcal{M}_{P B K+E I}(t 2)$}

When epigenetic factors are removed from $\mathcal{M}_{\mathrm{PBK}+\mathrm{EI}}$ and the model transformed into $\mathcal{M}_{\mathrm{PBK}}$, i.e., model = "t2", then the estimation of cpt values for $D K K 2$ remain the same as in model = "t1." The same computations apply for genes DKK3-1, DKK3 - 2, SFRP2, SFRP3, SFRP4, SFRP5, LEF1, $D A C T 1, D A C T 2$, and $D A C T 3$, in model = "t2."

Figure 8 shows the pictorial representation of one of the cpt in $\mathcal{M}_{\mathrm{PBK}+\mathrm{EI}}$ and $\mathcal{M}_{\mathrm{PBK}}$.

\subsubsection{DACT3 in $\mathcal{M}_{P B K+E I}(t 1)$}

The conditional probability value for a gene being active or inactive is estimated from generated frequency estimates that can help derive probability values. These frequencies depict the following cases: (a) gene repressed in normal (represented by vector offINn), (b) gene expressed in normal (represented by vector onINn), (c) gene repressed in tumorous (represented by vector offINt), and (d) gene expressed in tumorous (represented by vector onINt) cases. For every $j$ th entry in the vecTraining, if the label (labelTraining $(j)$ ) is normal $(\leq 0)$ and the DACT3 gene expression (vecTraining $(j)$ ) is less than the estimated median ( $\leq$ vecmedian), then the value in vecTraining $(j)$ is appended to offINn. Here, expression level lower than median indicates probable gene repression in the normal case. If the label (labelTraining $(j))$ is normal $(\leq 0)$ and the DACT3 gene expression (vecTraining $(j)$ ) is greater than the estimated median ( $\geq$ vecmedian), then the value in vecTraining $(j)$ is appended to onINn. Here, expression level greater than median indicates probable gene activation in the normal case. If the label (labelTraining $(j)$ ) is tumorous $(\geq 0)$ and the $D A C T 3$ gene expression (vecTraining $(j)$ ) is less than the estimated median ( $\leq$ vecmedian), then the value in vecTraining $(j)$ is appended to offINt. Here, expression level lower than median indicates probable gene repression in the tumor case. And finally, if the label (labelTraining $(j)$ ) is tumorous $(\geq 0)$ and the $D A C T 3$ gene expression (vecTraining $(j)$ ) is greater than the estimated median ( $\geq$ vecmedian), then the value in vecTraining $(j)$ is appended to onINt. Here, expression level greater than median indicates probable gene activation in the tumorous case.

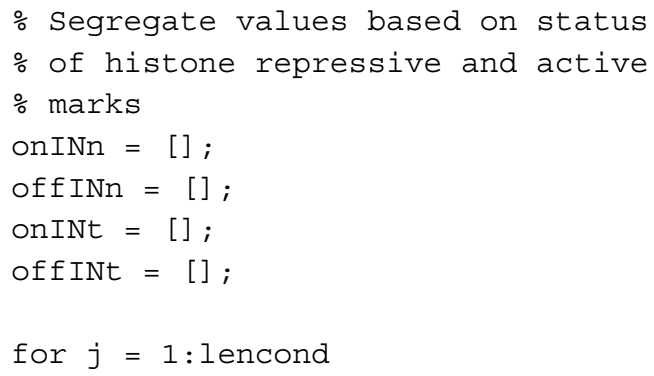




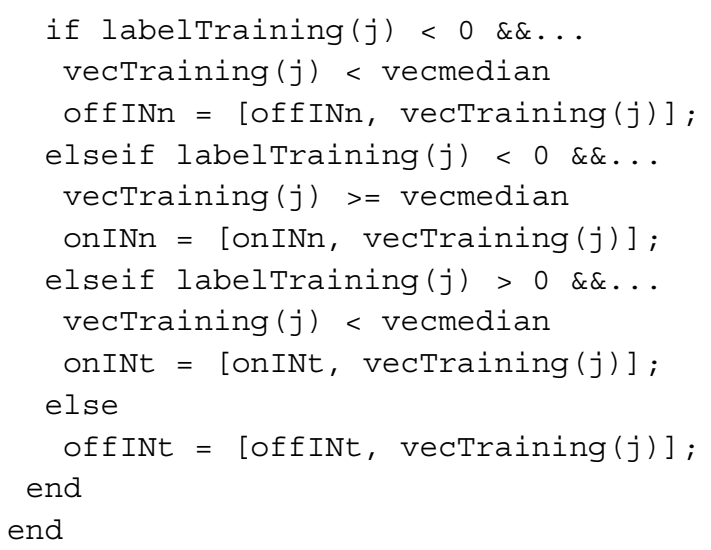

Lets again see how (1) the probability table would look like and (2) the probability table is stored in BNT [4], before estimating the values for cpt of DACT3. Table 14 represents the conditions of Sample, H3K4me3, and $H 3 K 4 m e 3$ the probable beliefs of events (DACT3 being on/off). Finally, from biological data presented in [2], the conditional probability values for the $D A C T 3$ gene being active based on the histone modification and the available samples suggest that $D A C T 3$ expression is high in normal samples when the histone repressive mark $H 3 K 27 m e 3$ is reduced and the activating mark $H 3 K 4 m e 3$ is present in high abundance. Thus, the probability, i.e., $\operatorname{Pr}(D A C T 3=$ active $\mid H K 327 m e 3=$ low, $H 3 K 4 m e 3=$ high, Sample $=$ normal) is the fraction of the number of 1's in the normal samples (a) and the total number of normal samples (A). For all other conditions of H3K27me3 and $H 3 K 4 m e 3$ when the Sample is normal, the probability of $D A C T 3$ being active is $(\mathrm{A}-\mathrm{a})$, i.e., flip or complementary of $\operatorname{Pr}(D A C T 3=$ active $\mid$ HK $327 m e 3=$ low, H3K4me3 = high, Sample = normal). This is because in all other conditions of the histone marks, the probability of $D A C T 3$ being active will be reverse of what it is when $H 3 K 27 m e 3$ is reduced and $H 3 K 4 m e 3$

Table 14 Conditional probability table for DACT3 in $\mathcal{M}_{\text {PBK }}$ (model - t1)

\begin{tabular}{lllll}
\hline \multicolumn{5}{l}{ CPT for DACT3 in $\boldsymbol{\mathcal { M }}_{\mathrm{PBK}+\text { El }}$ (model $-\mathrm{t}$ 1) } \\
\hline H3K27me3 & H3K4me3 & Sample & $\operatorname{Pr}($ DACT3=Off $)$ & $\operatorname{Pr}($ DACT3=On) \\
\hline 1 & 1 & Normal & $\mathrm{h}(1)$ & $\mathrm{I}(9)$ \\
2 & 1 & Normal & $\mathrm{h}(2)$ & $\mathrm{I}(10)$ \\
1 & 2 & Normal & $\mathrm{I}(3)$ & $\mathrm{h}(11)$ \\
2 & 2 & Normal & $\mathrm{h}(4)$ & $\mathrm{I}(12)$ \\
1 & 1 & Tumor & $\mathrm{h}(5)$ & $\mathrm{I}(13)$ \\
2 & 1 & Tumor & $\mathrm{h}(6)$ & $\mathrm{I}(14)$ \\
1 & 2 & Tumor & $\mathrm{I}(7)$ & $\mathrm{h}(15)$ \\
2 & 2 & Tumor & $\mathrm{h} \mathrm{(8)}$ & $\mathrm{I}(16)$ \\
\hline
\end{tabular}

h - probability of event being high; I - probability of event being low. 1 - low; 2 - high Serial numbers in brackets represent the ordering of numbers in vectorial format is present in abundance. Similarly, in case of tumorous samples, the probability of $D A C T 3$ being active will occur when $H 3 K 27 m e 3$ is reduced and H3K4me3 is high abundance (a rare phenomena). Thus, the probability, i.e., $\operatorname{Pr}(D A C T 3=$ active $\mid$ HK $327 m e 3=$ low, H3K $4 m e 3=$ high, Sample $=$ tumorous) is the fraction of the number of 1's in the tumorous sample (b) and the total number of tumorous samples (B). For all other conditions of $H 3 K 27 m e 3$ and $H 3 K 4 m e 3$ when the Sample is tumorous, the probability of $D A C T 3$ being active is (B-b), i.e., flip or complementary of $\operatorname{Pr}(D A C T 3=$ active $\mid H K 327 m e 3=$ low, H3K4me3 = high, Sample = tumorous). The reason for flip is the same as described above.

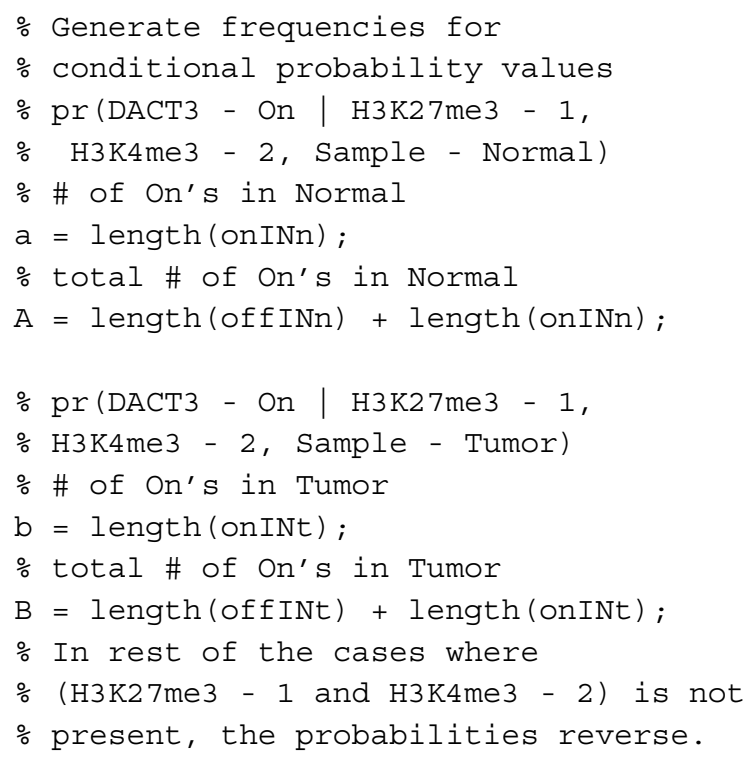

After the values in $\mathrm{T}$ have been established, a constant 1 is added as pseudo-count to convert the distribution to a probability distribution via Dirichlet process. Finally, the frequencies in $\mathrm{T}$ are normalized in order to obtain the final conditional probability values for DACT3. Figure 9 shows the pictorial representation of one of the cpt in $\mathcal{M}_{\mathrm{PBK}+\mathrm{EI}}$.

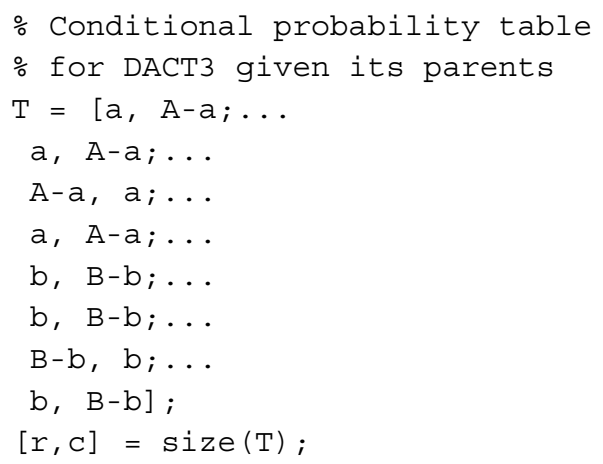

\% Convert the table to probability 


\begin{tabular}{|c|c|c|c|c|c|}
\hline & & & \multicolumn{3}{|c|}{$\begin{array}{l}\text { Conditional probability table for node } \\
\text { DACT } 3 \text { with parents sample } \\
\text { H3K27me3 and H3K4me3 (model }=+1)\end{array}$} \\
\hline & H3K27me & H3K4me3 & Sample & $\operatorname{Pr}(D A C T 3=0 f f)$ & $\operatorname{Pr}(D A C T 3=O n)$ \\
\hline DACT3 & low & low & Off & $(A-a+1) /(A+2)$ & $(a+1) /(A+2)$ \\
\hline & high & low & Off & $(A-a+1) /(A+2)$ & $(a+1) /(A+2)$ \\
\hline & low & high & Off & $(a+1) /(A+2)$ & $(A-a) /(A+2)$ \\
\hline & high & high & Off & $(A-a+1) /(A+2)$ & $(a+1) /(A+2)$ \\
\hline & low & low & On & $(b+1) /(B+2)$ & $(B-b+1) /(B+2)$ \\
\hline & high & low & On & $(b+1) /(B+2)$ & $(B-b+1) /(B+2)$ \\
\hline & low & high & On & $(B-b+1) /(B+2)$ & $(b+1) /(B+2)$ \\
\hline & high & high & On & $(b+1) /(B+2)$ & $(B-b+1) /(B+2)$ \\
\hline
\end{tabular}

Fig. 9 Conditional probability table for node DACT3 in $\mathcal{M}_{\mathrm{PBK}+\mathrm{El}}$

\% distribution via Dirichlet process

$\mathrm{T}=\mathrm{T}+1$;

for $i=1: r$

$T(i,:)=T(i,:) \cdot / \operatorname{sum}(T(i,:))$;

end

Finally, for every gene, after the computation of the probability values in their respective $\mathrm{cpt}$, the function generateGenecpd returns the following arguments as output.

gene_cpd = struct ( );

gene_cpd.vecmedian = vecmedian;

gene_cpd. $\mathrm{T}=\mathrm{T}$;

Tables 8 and 9 from [1] show the assumed and computed estimates for all the nodes that represent non-genetic and genetic factors in the modeled pathway driven by the dataset. It might be that the probability values deviate from the mathematics formulations as these formulations do not capture all the intricacies of the biological phenomena. For example, the cross talk that happens between the histone modifiers varies the expression of DACT3. But these time-varying dynamics cannot be captured in the model as the model represents a static time snapshot of the phenomena. More detailed explanation of this phenomena is available in [2]. The cpd for DACT3 in Table 9 states that when $H 3 K 27 m e 3$ is low and $H 3 K 4 m e 3$ is high, irrespective of the state of the sample, the belief represented by the conditional probability that $D A C T 3$ is repressed or off is high (and vice versa). Figure 9 shows the mathematical representation of the same. Similar interpretations can found for other cases.

\section{A programming project for practice}

To get a feel of the project, interested readers might want to implement the following steps when the evidence eviDence is "me." The code needs to be embedded as a case in the switch part of the twoHoldOutExp function. The idea is to perturb the methylation nodes with binary values and find if one can converge to the correct prediction of state of TRCMPLX as well as the Sample. These binary values are stored in a vector and represents a permutation of the methylation states of the methylation node in $\mathcal{M}_{\mathrm{PBK}+\mathrm{EI}}$. Varying the values of the vector can help study how perturbations affect the predictions from the network. The steps are given below:

1. Define variables for storing predictions of TRCMPLX (tempTRCMPLX) and Sample (tempsample).

2. Find the total number of methylation cases in $\mathcal{M}_{\text {PBK+EI }}$ and store the number in a variable noMethylation.

3. Generate binary values for noMethylation nodes. Define a cell (binarystatesofMethylation) that can store vectors of binary values where every permutation represents a set of methylation states. The total number of permutations should be $2^{\text {noMethylation }}$ (stored in noMethylationConfig).

One might want to use quantizer and num2bin functions from MATLAB. 
4. Next, generate methylation evidences. Define a 2D matrix variable methylationEvidence that stores the methylation evidences. One might want to use the MATLAB function str2num. Finally, add a value of 1 to methylationEvidence as the BNT takes in " 1 " and " 2 " as states representing binary values.

5. Build evidence for inference for every test example. The following steps might be necessary

- For every methylation configuration and for every methylation node, build evidence.

- Build a new Bayesian network in bnetEngine using jtree_inf_engine and store the modified engine (in engine) using the function enter_evidence.

- Finally, compute the $\operatorname{Pr}(T R C M P L X=2 \mid$ ge as evidence $)$ and $\operatorname{Pr}($ Sample $=2 \mid$ ge as evidence $)$ using the function marginal_nodes.

6. Store predicted results on observed methylation in structure Runs indexed with runCnt.

After the section of new code is filled in, run the code and check the results.

\section{Conclusions}

A pedagogical walkthrough of a computational modeling and simulation project is presented using parts of programming code interleaved with theory. The purpose behind this endeavor is to acclimatize and ease the understanding of beginner students and researchers in transition, who intend to work on computational signaling biology projects. To this end, static Bayesian network models for the Wnt signaling pathway have been selected for elucidation. Due to the paucity of available manuscripts that explain the computational experiments from a tutorial perspective because of unwanted restrictive policies, this endeavor is a small step in this direction.

\section{Acknowledgements}

Thanks to (1) all the anonymous reviewers and copy editors as well as editors at the Eurasip BSB for their effort in refining this manuscript; (2) Springer Eurasip Journal of Bioinformatics and Systems Biology for providing a waiver on the processing fee for this manuscript, without which it would not have been possible to process the manuscript; (3) The Royal Society of Chemistry (RSC) for giving permission to reproduce parts of material in [1]; (4) Netherlands Bioinformatics Centre (NBIC) for funding the project; (5) Dr. ir. R. H. J. Fastenau (Dean of Faculty of Electrical Engineering Mathematics and Computer Science), Dr. Prof. Ir. K. Ch. A. M. Luyben (Rector Magnificus), and Dr. D. J. van den Berg (President) at the Delft University of Technology, for providing support for conducting this work and giving permission to submit the manuscript; (6) Dr. Wim Verhaegh (faculty at Netherlands Bioinformatics Centre and a principal scientist at Molecular Diagnostic Lab in Philips Research) for providing technical details of Naive Bayes model for replicating experiments in [3]; (7) Dr. Marcel J. T. Reinders (scientific director of Bioinformatics Research at Netherlands Bioinformatics Centre and a professor at Delft University of Technology) for refining the proposed model and suggesting inclusion of methylation data; (8) Dr. Robert P.W. Duin (retired associate professor at Delft University of Technology) for informal discussions on 2-holdout experiments; and (9) Dr. Jeroen de Ridder (assistant professor at the Delft University of Technology) and (10) PhD candidate Marc Hulsman (Delft University of Technology), for informal discussions on estimation of probability values on methylation data. Finally, the author is indebted to Mr. Prabhat Sinha and Mrs. Rita Sinha for financially supporting this project while the author was on educational and work leave.

This + manuscript is a part of the Netherlands Bioinformatics Centre (NBIC) BioRange-II project BR2.5 (code - IGD71G). It complements and uses material from [1]. The Netherlands Bioinformatics Centre was supported by the Netherlands Genomics Initiative (NGI) via the Netherlands Genomics Institute in The Netherlands.

Shriprakash Sinha wrote this manuscript while working as an Independent Researcher.

\section{Competing interests}

The author declares that he has no competing interests.

Received: 12 June 2015 Accepted: 22 July 2016

Published online: 08 August 2016

\section{References}

1. S Sinha, Integration of prior biological knowledge and epigenetic information enhances the prediction accuracy of the Bayesian Wnt pathway. Integr. Biol. 6, 1034-1048 (2014). doi:10.1039/c4ib00124a

2. X Jiang, J Tan, J Li, S Kivimae, X Yang, L Zhuang, PL Lee, MTW Chan, L Stanton, ET Liu, BNR Cheyette, Q Yu, Dact3 is an epigenetic regulator of $\mathrm{Wnt} / \beta$-catenin signaling in colorectal cancer and is a therapeutic target of histone modifications. Cancer Cell. 13(6), 529-541 (2008)

3. W Verhaegh, P Hatzis, H Clevers, A van de Stolpe, Personalized cancer treatment selection using computational signaling pathway models. Cancer Res. San Antonio Breast Cancer Symp. 71(24), 524-525 (2011)

4. K Murphy, The Bayes net toolbox for MATLAB. Comput. Sci. Stat. 33(2), 1024-1034 (2001)

5. O Aguilera, M Fraga, E Ballestar, M Paz, M Herranz, J Espada, J Garcia, A Munoz, M Esteller, J Gonzalez-Sancho, Epigenetic inactivation of the Wnt antagonist dickkopf-1 (DKK-1) gene in human colorectal cancer. Oncogene. 25(29), 4116-4121 (2006)

6. H Suzuki, E Gabrielson, W Chen, R Anbazhagan, M Van Engeland, MP Weijenberg, JG Herman, SB Baylin, A genomic screen for genes upregulated by demethylation and histone deacetylase inhibition in human colorectal cancer. Nat. Genet. 31(2), 141-149 (2002)

7. G Yuan, C Wang, C Ma, N Chen, Q Tian, T Zhang, W Fu, Oncogenic function of DACT1 in colon cancer through the regulation of $\beta$-catenin. PloS ONE. 7(3), 34004 (2012)

8. H Clevers, $\mathrm{Wnt} /[\beta]$-catenin signaling in development and disease. Cell. 127(3), 469-480 (2006)

9. L Kriegl, D Horst, JA Reiche, J Engel, T Kirchner, A Jung, LEF-1 and TCF4 expression correlate inversely with survival in colorectal cancer. J. Transl. Med. 8,123 (2010)

10. T Gardner, J Faith, Reverse-engineering transcription control networks. Phys. Life Rev. 2, 65-88 (2005)

11. S Roehrig, Probabilistic inference and path analysis. Decis. Support. Syst. 16, 55-66 (1996)

12. J Pearl, Probabilistic reasoning in intelligent systems: networks of plausible inference. (Morgan Kaufmann, USA, 1988)

13. J Pearl, Causality: models, reasoning, and inference. (Cambridge Univ Pr, United Kingdom, 2000)

14. E Charniak, Bayesian networks without tears. Al Mag. 12, 50 (1991)

15. CJ Needham, JR Bradford, AJ Bulpitt, DR Westhead, A primer on learning in Bayesian networks for computational biology. PLoS Comput. Biol. 3, e129 (2007)

16. M Bayes, M Price, An essay towards solving a problem in the doctrine of chances. Phil. Trans. 53, 370 (1763)

17. N Friedman, M Linial, I Nachman, D Pe'er, Using Bayesian networks to analyze expression data. J. Comput. Biol. 7, 601-620 (2000)

18. A Hartemink, D Gifford, T Jaakkola, R Young, Using graphical models and genomic expression data to statistically validate models of genetic regulatory networks. Pac. Symp. Biocomput. 1825(1), 18-28 (2001) 
19. K Sachs, D Gifford, T Jaakkola, P Sorger, D Lauffenburger, Bayesian network approach to cell signaling pathway modeling. Science STKE. 148, 38-42 (2002)

20. K Sachs, O Perez, D Pe'er, D Lauffenburger, G Nolan, Causal protein-signaling networks derived from multiparameter single-cell data. Science. 308, 523 (2005)

21. D Pe'er, A Regev, G Elidan, N Friedman, Inferring subnetworks from perturbed expression profiles. Bioinformatics. 17, S215 (2001)

22. R Brent, L Lok, A fishing buddy for hypothesis generators. Science. $\mathbf{3 0 8}$, 504 (2005)

23. A Gregorieff, $\mathrm{H}$ Clevers, Wnt signaling in the intestinal epithelium: from endoderm to cancer. Genes Dev. 19(8), 877-890 (2005)

24. JF Costello, C Plass, Methylation matters. J. Med. Genet. 38(5), 285-303 (2001)

25. PM Das, R Singal, DNA methylation and cancer. J. Clinical Oncol. 22(22), 4632-4642 (2004)

26. JPJ Issa, DNA methylation as a therapeutic target in cancer. Clin. Cancer Res. 13(6), 1634-1637 (2007)

27. H Suzuki, DN Watkins, KW Jair, KE Schuebel, SD Markowitz, WD Chen, TP Pretlow, B Yang, Y Akiyama, M Van Engeland, Epigenetic inactivation of SFRP genes allows constitutive Wnt signaling in colorectal cancer. Nat. Genet. 36(4), 417-422 (2004)

28. C Niehrs, Function and biological roles of the dickkopf family of Wnt modulators. Oncogene. 25(57), 7469-7481 (2006)

29. H Sato, H Suzuki, M Toyota, M Nojima, R Maruyama, S Sasaki, H Takagi, Y Sogabe, Y Sasaki, M Idogawa, T Sonoda, M Mori, K Imai, T Tokino, Y Shinomura, Frequent epigenetic inactivation of dickkopf family genes in human gastrointestinal tumors. Carcinogenesis. 28(12), 2459-2466 (2007)

30. H Taniguchi, H Yamamoto, T Hirata, N Miyamoto, M Oki, K Nosho, Y Adachi, T Endo, K Imai, Y Shinomura, Frequent epigenetic inactivation of Wnt inhibitory factor- 1 in human gastrointestinal cancers. Oncogene. 24(53), 7946-7952 (2005)

31. BD Strahl, CD Allis, The language of covalent histone modifications. Nature. 403(6765), 41-45 (2000)

32. CL Peterson, MA Laniel, Histones and histone modifications. Curr. Biol. 14(14), 546-551 (2004)

33. ML Waterman, Lymphoid enhancer factor/t cell factor expression in colorectal cancer. Cancer Metastasis Rev. 23(1), 41-52 (2004)

34. GS Yochum, Multiple Wnt/ $\beta$-catenin responsive enhancers align with the myc promoter through long-range chromatin loops. PloS ONE. 6(4), 18966 (2011)

35. KM Schmidt-Ott, TNH Masckauchan, X Chen, BJ Hirsh, A Sarkar, J Yang, N Paragas, VA Wallace, D Dufort, P Pavlidis, $\beta$-catenin/TCF/LEF controls a differentiation-associated transcriptional program in renal epithelial progenitors. Development. 134(17), 3177-3190 (2007)

36. SS Kanwar, Y Yu, J Nautiyal, BB Patel, APN Majumdar, The Wnt/ $\beta$-catenin pathway regulates growth and maintenance of colonospheres. Mol. Cancer. 9(1), 212 (2010)

37. G Caldwell, C Jones, P Taniere, R Warrack, Y Soon, G Matthews, D Morton, The Wnt antagonist SFRP1 is downregulated in premalignant large bowel adenomas. Br. J. Cancer. 94(6), 922-927 (2006)

38. N Reguart, B He, Z Xu, L You, AY Lee, J Mazieres, I Mikami, S Batra, R Rosell, F McCormick, Cloning and characterization of the promoter of human Wnt inhibitory factor-1. Biochem. Biophys. Res. Commun. 323(1), 229-234 (2004)

39. JM Gonzalez-Sancho, O Aguilera, JM Garcia, N Pendas-Franco, C Pena, S Cal, AG deHerreros, F Bonilla, A Munoz, The Wnt antagonist dickkopf-1 gene is a downstream target of $\beta$-catenin/TCF and is downregulated in human colon cancer. Oncogene. 24(6), 1098-1103 (2004)

40. N Pendas-Franco, J Garcia, C Pena, N Valle, H Palmer, M Heinaniemi, C Carlberg, B Jimenez, F Bonilla, A Munoz, Dickkopf-4 is induced by $\mathrm{tcf} / \beta$-catenin and upregulated in human colon cancer, promotes tumour cell invasion and angiogenesis and is repressed by $1 \alpha$, 25-dihydroxyvitamin d3. Oncogene. 27(32), 4467-4477 (2008)

41. S Baehs, A Herbst, SE Thieme, C Perschl, A Behrens, S Scheel, A Jung, T Brabletz, B Goke, H Blum, Dickkopf-4 is frequently down-regulated and inhibits growth of colorectal cancer cells. Cancer Lett. 276(2), 152-159 (2009)
42. A Matsui, T Yamaguchi, S Maekawa, C Miyazaki, S Takano, T Uetake, T Inoue, M Otaka, H Otsuka, T Sato, Dickkopf-4 and-2 genes are upregulated in human colorectal cancer. Cancer Sci. 100(10), 1923-1930 (2009)

43. M Zitt, G Untergasser, A Amberger, P Moser, S Stadlmann, M Zitt, HM Muller, G Muhlmann, A Perathoner, R Margreiter, Dickkopf-3 as a new potential marker for neoangiogenesis in colorectal cancer: expression in cancer tissue and adjacent non-cancerous tissue. Dis. Markers. 24(2), 101-109 (2008)

44. J Veeck, E Dahl, Targeting the Wnt pathway in cancer: the emerging role of dickkopf-3. Biochimica et Biophysica Acta (BBA)-Reviews Cancer. 6, 422-433 (2012)

45. PD Brophy, KM Lang, GR Dressler, The secreted frizzled related protein 2 (SFRP2) gene is a target of the PAX2 transcription factor. J. Biol. Chem. 278(52), 52401-52405 (2003)

46. Q Feng Han, W Zhao, J Bentel, AM Shearwood, N Zeps, D Joseph, B lacopetta, A Dharmarajan, Expression of SFRP-4 and $\beta$-catenin in human colorectal carcinoma. Cancer Lett. 231(1), 129-137 (2006)

47. D Huang, B Yu, Y Deng, W Sheng, Z Peng, W Qin, X Du, Sfrp4 was overexpressed in colorectal carcinoma. J. Cancer Res. Clin. Oncol. 136(3), 395-401 (2010)

48. B Hoang, M Moos, S Vukicevic, FP Luyten, Primary structure and tissue distribution of FRZB, a novel protein related to drosophila frizzled, suggest a role in skeletal morphogenesis. J. Biol. Chem. 271(42) 26131-26137 (1996)

49. S Kivimae, XY Yang, BNR Cheyette, All DACT (dapper/frodo) scaffold proteins dimerize and exhibit conserved interactions with vangl, dvl, and serine/threonine kinases. BMC Biochemistry. 12(1), 33 (2011)

50. E Charniak, Bayesian networks without tears. Al Mag. 12(4), 50 (1991)

\section{Submit your manuscript to a SpringerOpen ${ }^{\circ}$ journal and benefit from:}

- Convenient online submission

- Rigorous peer review

- Immediate publication on acceptance

- Open access: articles freely available online

- High visibility within the field

- Retaining the copyright to your article

Submit your next manuscript at springeropen.com 